\title{
Almost sure convergence for stochastically biased random walks on trees
}

\author{
by \\ Gabriel Faraud, Yueyun Hu and Zhan Shi \\ Université Paris XIII, Université Paris XIII $\mathcal{E}$ Université Paris VI
}

\begin{abstract}
Summary. We are interested in the biased random walk on a supercritical Galton-Watson tree in the sense of Lyons 22 and Lyons, Pemantle and Peres [28, and study a phenomenon of slow movement. In order to observe such a slow movement, the bias needs to be random; the resulting random walk is then a tree-valued random walk in random environment. We investigate the recurrent case, and prove, under suitable general integrability assumptions, that upon the system's non-extinction, the maximal displacement of the walk in the first $n$ steps, divided by $(\log n)^{3}$, converges almost surely to a known positive constant.
\end{abstract}

Keywords. Biased random walk on a Galton-Watson tree, branching random walk, slow movement, random walk in a random environment.

2010 Mathematics Subject Classification. 60J80, 60G50, 60K37.

\section{Introduction}

\subsection{Stochastically biased random walks on Galton-Watson trees}

Let $\mathbb{T}$ be a supercritical Galton-Watson tree rooted at $\varnothing$. Two vertices $x$ and $y$ are said to be connected, and denoted by $x \sim y$, if $x$ is either the parent or a child of $y$. For a vertex $x \in \mathbb{T}$, we denote by $|x|$ the distance between $x$ and the root $\varnothing$, and $\varnothing=x_{0}, x_{1}, \ldots, x_{|x|}$ the shortest path between the root and $x$. Let $\omega=(\omega(x), x \in \mathbb{T} \backslash\{\varnothing\})$ be a sequence 
of vectors defined by $\omega(x)=(\omega(x, y), y \sim x)$ such that $\omega(x, y)>0, \forall y \sim x$ and that $\sum_{y \sim x} \omega(x, y)=1$.

Given the sequence $\omega$, we define a random walk $\left(X_{n}, n \geq 0\right)$ on $\mathbb{T}$ whose transition probabilities are

$$
P_{\omega}\left(X_{n+1}=y \mid X_{n}=x\right)=\omega(x, y) .
$$

For each vertex $x \in \mathbb{T} \backslash\{\varnothing\}$, we denote its parent by $\overleftarrow{x}$, and its children by $\left(x^{(1)}, \cdots, x^{(N(x))}\right)$, where $N(x)$ stands for the number of children of $x$. Instead of looking at $\omega(x, y)$ (for $y \sim x$ and $x \in \mathbb{T})$, it is often more convenient to study $A(x):=\left(A_{i}(x), 1 \leq i \leq N(x)\right)$ defined by

$$
A_{i}(x):=\frac{\omega\left(x, x^{(i)}\right)}{\omega(x, \overleftarrow{x})}, \quad 1 \leq i \leq N(x)
$$

Example (Biased random walk on a Galton-Watson tree). When $A_{i}(x) \equiv \lambda, \forall x$, $\forall i$ (where $0<\lambda<\infty$ is a constant), the random walk $\left(X_{n}\right)$ is the $\lambda$-biased random walk on $\mathbb{T}$ studied by Lyons [22], [23], Lyons, Pemantle and Peres [27], 28], Peres and Zeitouni [32], and Ben Arous et al. [3]. More particularly, if $A_{i}(x) \equiv 1, \forall x, \forall i$, we get the simple random walk on $\mathbb{T}$.

Ben Arous and Hammond [4] considered the case that $A_{i}(x)$ does not depend on $x$ nor on $i$, but can be random. They called the resulting walk $\left(X_{n}\right)$ randomly biased walk on $\mathbb{T}$, and proved that the walk is more regular in some sense than the biased random walk.

We focus, in this paper, on a phenomenon of slow movement of the walk in the recurrent case. In order to exhibit the slow movement, the transition probabilities need to be random (which was already the case in the aforementioned work of Ben Arous and Hammond [4]): the resulting random walk $\left(X_{n}\right)$ is a so-called random walk in random environment. In dimension 1 (which, informally, corresponds to the case $N(x)=1$ for all $x$ ), a celebrated theorem of Sinai [33] says that $\frac{X_{n}}{(\log n)^{2}}$ converges in distribution to a non-degenerate law.

From now on, we assume $(A(x), x \in \mathbb{T} \backslash\{\varnothing\})$ to be i.i.d., and write $A=\left(A_{1}, \cdots, A_{N}\right)$ for a random vector having the distribution of any of $A(x)$. Note that here $N$ itself is random and follows the law of reproduction of $\mathbb{T}$. We always use $\mathbf{P}$ to denote the probability with respect to the environment, and $\mathbb{P}:=\mathbf{P} \otimes P_{\omega}$ the annealed measure. It is convenient to consider $(\omega, \mathbb{T})$ as a marked tree, see (1.6) below in terms of a branching random walk. As such, when we say "for almost all environment $\omega$ ", we mean, in fact, for almost all $(\omega, \mathbb{T})$.

Let us introduce the logarithmic moment-generating function of $A$ :

$$
\psi(t):=\log \mathbf{E}\left\{\sum_{i=1}^{N} A_{i}^{t}\right\} \in(-\infty, \infty], \quad t \geq 0 .
$$


In particular, $\psi(0)=\log \mathbf{E}(N)$. We always assume $\psi(0)>0$, so that the Galton-Watson tree is supercritical. Furthermore, we assume that there exists some $\delta>0$ such that

$$
\psi(t)<\infty, \quad \forall t \in(-\delta, 1+\delta), \text { and that } \mathbf{E}\left(N^{1+\delta}\right)<\infty .
$$

We first recall the following recurrence/transience criterion:

Theorem A (Lyons and Pemantle [25]). (i) If $\inf _{t \in[0,1]} \psi(t) \leq 0$, then for almost all $\omega$, $\left(X_{n}\right)$ is recurrent.

(ii) If $\inf _{t \in[0,1]} \psi(t)>0$, then for almost all $\omega,\left(X_{n}\right)$ is transient on the set of nonextinction.

Theorem A was proved in [25] under the additional condition that the distribution of $A_{i}$ does not depend on $i$; this condition was removed in Faraud [11]. See also Menshikov and Petritis [30] for a proof of this criterion (under the additional assumptions that $N>1$ is deterministic and that the law of $A_{i}$ does not depend on $i$ ) via Mandelbrot's multiplicative cascades.

The transient case (i.e., if $\inf _{t \in[0,1]} \psi(t)>0$ ) has received much research attention recently ([1], [2], [3], [4]).

If $\inf _{t \in[0,1]} \psi(t)<0$, the walk $\left(X_{n}\right)$ is positive recurrent for almost all $\omega$; in this case, it is not hard (see [17], under the additional assumptions that $N$ is deterministic and that the law of $A_{i}$ does not depend on $i$ ) to prove that $\frac{1}{\log n} \max _{0 \leq k \leq n}\left|X_{k}\right|$ converges almost surely to a positive constant.

We assume $\inf _{t \in[0,1]} \psi(t)=0$ from now on. There are two different regimes in this case, depending on the sign of $\psi^{\prime}(1)=\mathrm{e}^{-\psi(1)} \mathbf{E}\left\{\sum_{i=1}^{N} A_{i} \log A_{i}\right\}$. If $\psi^{\prime}(1)<0$ (and $\inf _{t \in[0,1]} \psi(t)=$ 0 ), then by defining $\kappa:=\inf \{t>1: \psi(t)=0\} \in(1, \infty]$ (with inf $\varnothing:=\infty$ ) and $\kappa_{1}:=$ $1-\frac{1}{\min \{\kappa, 2\}} \in\left(0, \frac{1}{2}\right]$, the order of magnitude of $\left|X_{n}\right|$ is, loosely speaking, $n^{\kappa_{1}}$. [That $\kappa>1$ is a consequence of the convexity of $\psi$.] More precisely, as far as strong convergence is concerned, Hu and Shi [17] proved (assuming $N$ is deterministic and that the law of $A_{i}$ does not depend on $i$ ) that $\max _{0 \leq k \leq n}\left|X_{k}\right|=n^{\kappa_{1}+o(1)}, \mathbb{P}$-almost surely. For (functional) weak convergence, Peres and Zeitouni [32] established a quenched functional central limit theorem for biased random walk on $\mathbb{T}$ (corresponding to the case $A_{i}=\frac{1}{\mathbf{E}(N)}, \forall i$, and thus $\kappa=\infty$; assuming moreover $N>1$ a.s.). The latter was extended by Faraud [1] for walks satisfying $\kappa \in(8, \infty]$ for the quenched case and $\kappa \in(5, \infty]$ for the annealed case. The problem of whether $\frac{\left|X_{n}\right|}{n^{\kappa} 1}$ converges weakly (in either quenched or annealed setting) for the whole region $\kappa \in(2, \infty]$ remains open, to the best of our knowledge. 

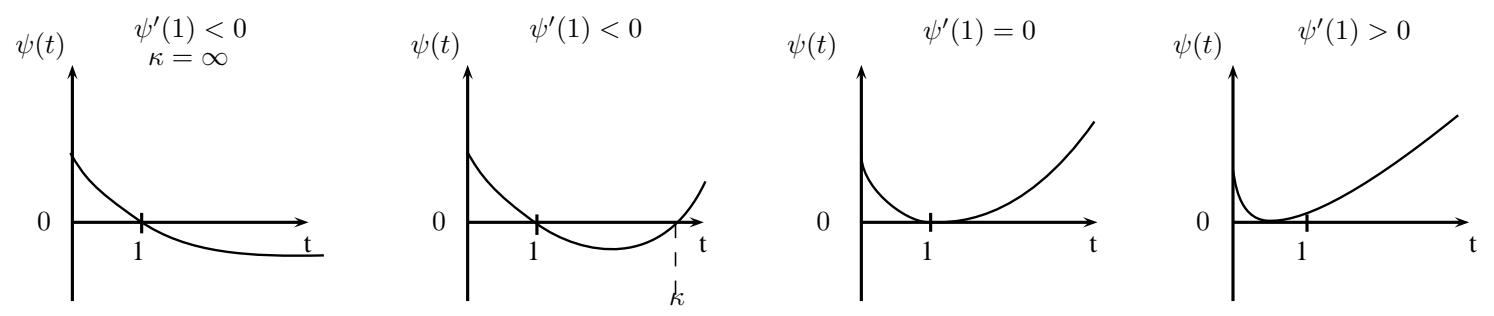

Figure 1: The different possible shapes for $\psi$

This paper is devoted to the study of the second situation: $\psi^{\prime}(1) \geq 0$ (and $\inf _{t \in[0,1]} \psi(t)=$ $0)$. It was known that the walk is then extremely slow, at least under the additional conditions that $N$ is deterministic and that the law of $A_{i}$ does not depend on $i$ : there exist constants $0<c_{1} \leq c_{2}<\infty$ such that (see [18])

$$
c_{1} \leq \liminf _{n \rightarrow \infty} \frac{\max _{0 \leq k \leq n}\left|X_{k}\right|}{(\log n)^{3}} \leq \limsup _{n \rightarrow \infty} \frac{\max _{0 \leq k \leq n}\left|X_{k}\right|}{(\log n)^{3}} \leq c_{2}, \quad \mathbb{P} \text {-a.s. }
$$

One of the main goals of this paper is to prove that almost sure convergence holds in (1.3) if $\psi^{\prime}(1) \geq 0$ (and $\inf _{t \in[0,1]} \psi(t)=0$ ). The limiting constant in (1.3), however, will have different natures depending on whether $\psi^{\prime}(1)=0$ or $\psi^{\prime}(1)>0$. If $\psi^{\prime}(1) \geq 0$ (and $\left.\inf _{t \in[0,1]} \psi(t)=0\right)$, there exists $0<\theta \leq 1$ such that

$$
\psi^{\prime}(\theta)=0 .
$$

The case $\theta=1$ corresponds to $\psi^{\prime}(1)=0$; in this case, the condition $\inf _{t \in[0,1]} \psi(t)=0$ is equivalent to $\psi(1)=0$, and the walk is null recurrent. The case $\theta<1$, on the other hand, corresponds to $\psi^{\prime}(1)>0$, and the walk is positive recurrent (see [1]).

To give the limiting constant in (1.3), we define

$$
\alpha_{\theta}:=\frac{3 \pi^{2}}{2} \frac{1}{\theta} \mathbf{E}\left(\sum_{i=1}^{N} A_{i}^{\theta}\left(\log A_{i}\right)^{2}\right)
$$

We write simply $\alpha$ for $\alpha_{1}$.

Theorem 1.1 Assume $\psi(1)=\psi^{\prime}(1)=0$. On the set of non-extinction,

$$
\lim _{n \rightarrow \infty} \frac{\max _{0 \leq k \leq n}\left|X_{k}\right|}{(\log n)^{3}}=\frac{4}{\alpha}, \quad \mathbb{P} \text {-a.s. }
$$

where the constant $\alpha=\alpha_{1}$ is defined in (1.5). 
Theorem 1.2 Assume $\inf _{t \in[0,1]} \psi(t)=0$ and $\psi^{\prime}(1)>0$. On the set of non-extinction,

$$
\lim _{n \rightarrow \infty} \frac{\max _{0 \leq k \leq n}\left|X_{k}\right|}{(\log n)^{3}}=\frac{1}{\alpha_{\theta}}, \quad \mathbb{P} \text {-a.s. }
$$

where $\theta \in(0,1)$ is as in (1.4) and $\alpha_{\theta}$ is defined in (1.5).

Theorem 1.2 is proved in Section 4, whereas the proofs of the upper and lower bounds in Theorem 1.1 are in Sections 5 and 6, respectively. In the next paragraph, we explain how it is that the $(\log n)^{3}$ rate in Theorem 1.1 arises, by means of an associated branching random walk which plays the role of potential for our walk $\left(X_{n}\right)$.

\subsection{Branching random walks and maxima along rays}

The influence of the random environment on the behaviour of $\left(X_{n}\right)$ is best formulated in terms of an associated potential process. To make the presentation easier, we artificially

add a special vertex, $\overleftarrow{\varnothing}$, which is to be thought of as the parent of $\varnothing$. Since the values of the transition probabilities at a finite number of vertices have no influence on any of the results of the paper, we feel free to modify the value of $\omega(\varnothing, \bullet)$, the transition probability at $\varnothing$, in such a way that $\left(A_{i}(x), 1 \leq i \leq N(x)\right)$, for $x \in \mathbb{T}$ (including $x=\varnothing$ now), form an i.i.d. collection of random variables. Let $\omega(\overleftarrow{\varnothing}, \varnothing)=1$, and define consistently

$$
\omega(\varnothing, \overleftarrow{\varnothing}):=\frac{1}{1+\sum_{i=1}^{N(\varnothing)} A_{i}(\varnothing)}
$$

The potential process associated with the random environment is defined by $V(\varnothing):=0$ and

$$
V(x):=-\sum_{y \in \rrbracket \varnothing, x \rrbracket} \log \frac{\omega(\overleftarrow{y}, y)}{\omega(\overleftarrow{y}, \overleftarrow{y})}, \quad x \in \mathbb{T} \backslash\{\varnothing\},
$$

where $\overleftarrow{y}$ is the parent of $\overleftarrow{y}$, $\varnothing, x \rrbracket$ the set of vertices on the shortest path connecting $\varnothing$ to $x$, and $\rrbracket \varnothing, x \rrbracket:=\llbracket \varnothing, x \rrbracket \backslash\{\varnothing\}$.

Clearly, $(V(x), x \in \mathbb{T})$ is a branching random walk, in the usual sense of Biggins [6]. It can be described as follows: Initially, a single particle is located at 0 , which is the ancestor of the system. At time 1, the ancestor dies, giving birth to new particles who form the first generation, and who are positioned according to the distribution of $\left(-\log A_{i}(\varnothing), 1 \leq\right.$ $i \leq N(\varnothing)$ ). At time 2, each of the particles in the first generation dies, giving birth to 
new particles that are positioned (with respect to their birth places) according to the same distribution of $\left(-\log A_{i}(\varnothing), 1 \leq i \leq N(\varnothing)\right)$; these new particles form the second generation. The system goes on according to the same mechanism. We assume that for any $n$, each particle at generation $n$ produces new particles independently of each other and of everything up to the $n$-th generation. The positions of the particles in the $n$-th generation are denoted by $(V(x),|x|=n)$.

The condition $\inf _{t \in[0,1]} \psi(t)=0$ is equivalent to $\inf _{t \in[0,1]} \mathbf{E}\left(\sum_{|x|=1} \mathrm{e}^{-t V(x)}\right)=1$, whereas $\psi^{\prime}(1) \geq 0$ means $\mathbf{E}\left(\sum_{|x|=1} V(x) \mathrm{e}^{-V(x)}\right) \leq 0$.

In the recurrent case, there is a simple relationship between the potential $(V(x), x \in \mathbb{T})$ and the walk $\left(X_{n}\right)$. For any $k \geq 0$, let

$$
\tau_{k}:=\inf \left\{j \geq 1:\left|X_{j}\right|=k\right\}, \quad \inf \varnothing:=\infty .
$$

So $\tau_{0}$ is the first return time to the root if the walk starts from $\varnothing$. It turns out that there exists $0<c(\omega)<\infty$ possibly depending on the environment, such that for any $n \geq 1$,

$$
\varrho_{n}:=P_{\omega}\left\{\tau_{n}<\tau_{0}\right\} \geq \frac{c(\omega)}{n} \exp \left(-\min _{|x|=n} \bar{V}(x)\right),
$$

where, for any vertex $x$, we write

$$
\bar{V}(x):=\max _{y \in \rrbracket \varnothing, x \rrbracket} V(y) .
$$

Inequality (1.7) was proved in [18] under the additional conditions that $N$ is deterministic and that the law of $A_{i}$ does not depend on $i$. Since the proof is simple, we reproduce it here: For any $x \in \mathbb{T}$, let $T(x):=\inf \left\{i \geq 0: X_{i}=x\right\}$ be the first hitting time of the walk at vertex $x$. By definition, for any $n \geq 1, \tau_{n}=\min _{|x|=n} T(x)$, so that

$$
P_{\omega}\left\{\tau_{n}<\tau_{0}\right\} \geq \max _{|x|=n} P_{\omega}\left\{T(x)<\tau_{0}\right\}
$$

We fix a vertex $x$ with $|x|=n$. To compute $P_{\omega}\left\{T(x)<\tau_{0}\right\}$, we define a random sequence $\left(\sigma_{j}\right)_{j \geq 0}$ (depending on $x$ ) by $\sigma_{0}:=0$ and

$$
\sigma_{j}:=\inf \left\{k>\sigma_{j-1}: X_{k} \in \llbracket \varnothing, x \rrbracket \backslash\left\{X_{\sigma_{j-1}}\right\}\right\}, \quad j \geq 1 .
$$

If the walk $\left(X_{n}\right)$ is recurrent, then $\left(\sigma_{j}\right)$ is well-defined.

Let $Z_{k}:=X_{\sigma_{k}}, k \geq 0$, which is the restriction of $\left(X_{j}\right)$ on the path $\llbracket \varnothing, x \rrbracket$. For $i \leq n$, let $x_{i}$ be the unique vertex in $\llbracket \varnothing, x \rrbracket$ with $\left|x_{i}\right|=i$ (in particular, $x_{0}=\varnothing, x_{n}=x$ ). Then for $1 \leq i<n$

$$
P_{\omega}\left\{Z_{k+1}=x_{i+1} \mid Z_{k}=x_{i}\right\}=\frac{\omega\left(x_{i}, x_{i+1}\right)}{\omega\left(x_{i}, x_{i+1}\right)+\omega\left(x_{i}, x_{i-1}\right)}=1-P_{\omega}\left\{Z_{k+1}=x_{i-1} \mid Z_{k}=x_{i}\right\},
$$


which yields

$$
\begin{aligned}
P_{\omega}\left\{T(x)<\tau_{0}\right\} & =\omega\left(\varnothing, x_{1}\right) P_{\omega}\left\{\left(Z_{k}\right) \text { hits } x \text { before hitting } \varnothing \mid Z_{0}=x_{1}\right\} \\
& =\frac{\omega\left(\varnothing, x_{1}\right) \mathrm{e}^{V\left(x_{1}\right)}}{\sum_{z \in \rrbracket \varnothing, x \rrbracket} \mathrm{e}^{V(z)}},
\end{aligned}
$$

the second identity following from a general formula (Zeitouni [34], formula (2.1.4)) for the exit problem of one-dimensional random walk in random environment. Since $\sum_{z \in \rrbracket \varnothing, x \rrbracket} \mathrm{e}^{V(z)} \geq$ $\max _{z \in \rrbracket \varnothing, x \rrbracket} \mathrm{e}^{V(z)}=\mathrm{e}^{\bar{V}(x)}$, this implies

$$
P_{\omega}\left\{T(x)<\tau_{0}\right\} \leq \omega\left(\varnothing, x_{1}\right) \mathrm{e}^{V\left(x_{1}\right)-\bar{V}(x)}
$$

Going back to (1.9), we immediately obtain (1.7) with $c(\omega):=\min _{|x|=1}\left[\omega(\varnothing, x) \mathrm{e}^{V(x)}\right]>0$.

The probability $\varrho_{n}$ is closely related to the maximal displacement of the branching random walk. The following simple observation was implicitly stated in [18] (pp. 1993-1996):

Fact 1.3 Assume $\inf _{t \in[0,1]} \psi(t)=0$ and $\psi^{\prime}(1) \geq 0$. Let $0<c<\infty$ be a constant. Almost surely on the set of non-extinction,

(i) if $\varrho_{n} \geq \mathrm{e}^{-(c+o(1)) n^{1 / 3}}$ for all sufficiently large $n$, then

$$
\liminf _{n \rightarrow \infty} \frac{1}{(\log n)^{3}} \max _{0 \leq k \leq n}\left|X_{k}\right| \geq \frac{1}{c^{3}}
$$

(ii) if $\varrho_{n} \leq \mathrm{e}^{-(c+o(1)) n^{1 / 3}}$ for all sufficiently large $n$, then

$$
\limsup _{n \rightarrow \infty} \frac{1}{(\log n)^{3}} \max _{0 \leq k \leq n}\left|X_{k}\right| \leq \frac{1}{c^{3}} .
$$

As such, an upper bound for $\min _{|x|=n} \bar{V}(x)$ yields, via inequality (1.7), a lower bound for $\varrho_{n}$, which, in turn, will lead to a lower bound for the maximal displacement of the walk $\left(X_{j}\right)$.

Theorem 1.4 Assume $\inf _{t \in[0,1]} \mathbf{E}\left\{\sum_{|x|=1} \mathrm{e}^{-t V(x)}\right\}=1$ and $\mathbf{E}\left\{\sum_{|x|=1} V(x) \mathrm{e}^{-V(x)}\right\} \leq 0$. Let $\theta \in(0,1]$ be such that $\mathbf{E}\left\{\sum_{|x|=1} V(x) \mathrm{e}^{-\theta V(x)}\right\}=0$. We have, on the set of non-extinction,

$$
\lim _{n \rightarrow \infty} \frac{1}{n^{1 / 3}} \min _{|x|=n} \bar{V}(x)=\left(\frac{3 \pi^{2} \sigma_{\theta}^{2}}{2}\right)^{1 / 3}, \quad \text { P-a.s. }
$$

where

$$
\sigma_{\theta}^{2}:=\frac{1}{\theta} \mathbf{E}\left\{\sum_{|x|=1} V(x)^{2} \mathrm{e}^{-\theta V(x)}\right\}
$$


We mention that Fang and Zeitouni [10] have independently obtained Theorem 1.4, under the condition that $N$ is non-random and $A_{i}(\varnothing)$, for $1 \leq i \leq N$, are i.i.d.

Comparing Theorem 1.4 with Theorems 1.1 and 1.2, we observe that (1.7) is optimal in the case $\psi^{\prime}(1)>0$ (or, equivalently, $\mathbf{E}\left\{\sum_{|x|=1} V(x) \mathrm{e}^{-V(x)}\right\}<0$ ), but not in the case $\psi^{\prime}(1)=0$ (or, equivalently, $\mathbf{E}\left\{\sum_{|x|=1} V(x) \mathrm{e}^{-V(x)}\right\}=0$ ).

The proofs of the theorems are organized as follows.

- Section 2, Theorem 1.4.

- Section 4. Theorem 1.2.

- Section 5. Theorem 1.1, upper bound.

- Section 6: Theorem 1.1, lower bound. [This is the heart of the paper.]

Section 3 is devoted to a probability estimate for one-dimensional random walks, which will be exploited in the proofs of Theorems 1.1 and 1.2 later on.

Throughout the paper, we use the convention $\sum_{\varnothing}:=0, \max _{\varnothing}:=0$ and $\min _{\varnothing}:=\infty$. The letter $c$, with or without subscript, denotes a finite and positive constant, whose value may vary from line to line. Furthermore, $a_{n} \sim b_{n}, n \rightarrow \infty$, means $\lim _{n \rightarrow \infty} \frac{a_{n}}{b_{n}}=1$.

\section{Proof of Theorem 1.4}

Assume $\psi(1)=0$, i.e., $\mathbf{E}\left\{\sum_{|x|=1} \mathrm{e}^{-V(x)}\right\}=1$.

The condition $\mathbf{E}\left(N^{1+\delta}\right)<\infty$ in (1.2) guarantees that $\mathbf{P}\{N(x)<\infty, \forall x\}=1(N(x)$ being the number of children of $x$ ). Recall that given a vertex $x \in \mathbb{T}, x_{0}:=\varnothing, x_{1}, \cdots$, $x_{|x|}:=x$ are the vertices on $\llbracket \varnothing, x \rrbracket$ with $\left|x_{i}\right|=i$ for any $0 \leq i \leq|x|$. The condition $\psi(1)=0$ yields that for any $n \geq 1$ and any measurable function $F: \mathbb{R}^{n} \times \mathbb{R}^{n} \rightarrow[0, \infty)$,

$$
\mathbf{E}\left(\sum_{|x|=n} \mathrm{e}^{-V(x)} F\left[V\left(x_{i}\right), N\left(x_{i-1}\right), 1 \leq i \leq n\right]\right)=\mathbf{E}\left(F\left[S_{i}, \nu_{i-1}, 1 \leq i \leq n\right]\right),
$$

where $\left(S_{i}-S_{i-1}, \nu_{i-1}\right)$, for $i \geq 1$, are i.i.d. random vectors, whose common distribution is determined by

$$
\mathbf{E}\left[f\left(S_{1}, \nu_{0}\right)\right]=\mathbf{E}\left(\sum_{|x|=1} \mathrm{e}^{-V(x)} f(V(x), N(\varnothing))\right)=\mathbf{E}\left(\sum_{i=1}^{N} A_{i} f\left(-\log A_{i}, N\right)\right),
$$

for any measurable function $f: \mathbb{R}^{2} \rightarrow[0, \infty)$. Considering only the first argument, (2.1) says that for any $n \geq 1$ and any measurable function $F: \mathbb{R}^{n} \rightarrow[0, \infty)$,

$$
\mathbf{E}\left(\sum_{|x|=n} \mathrm{e}^{-V(x)} F\left(V\left(x_{i}\right), 1 \leq i \leq n\right)\right)=\mathbf{E}\left[F\left(S_{i}, 1 \leq i \leq n\right)\right]
$$


with the distribution of $S_{1}$ determined by

$$
\mathbf{E}\left(f\left(S_{1}\right)\right)=\mathbf{E}\left(\sum_{|x|=1} \mathrm{e}^{-V(x)} f(V(x))\right)
$$

for any measurable function $f: \mathbb{R} \rightarrow[0, \infty)$. Formula (2.3) is well-known, and can be proved by means of a simple argument by induction in $n$. See, for example, Biggins and Kyprianou [7]. The proof of (2.1) follows exactly from the same argument. In Section [6, we will see an extension of (2.1), which, in particular, gives a probabilistic interpretation of the new random walk $\left(S_{i}\right)$.

[The distribution of $S_{1}$ is well-defined upon the assumption $\psi(1)=0$. If furthermore $\psi^{\prime}(1)=0$, then $\mathbf{E}\left(S_{1}\right)=0$; in words, $\left(S_{n}\right)$ is a mean-zero random walk, with $\sigma^{2}=\mathbf{E}\left(S_{1}^{2}\right)$.]

Formula (2.3) naturally leads to studying the one-dimensional random walk $\left(S_{n}\right)$. However, we sometimes need to work in a slightly more general setting: For each $n \geq 1$, let $X_{i}^{(n)}, 1 \leq i \leq n$, be i.i.d. real-valued variables; define $S_{0}^{(n)}:=0$ and $S_{j}^{(n)}:=\sum_{i=1}^{j} X_{i}^{(n)}$ for $1 \leq j \leq n$. Let $\left(a_{n}\right)$ be positive numbers such that $a_{n} \rightarrow \infty$ and $\frac{a_{n}^{2}}{n} \rightarrow 0, n \rightarrow \infty$. Assume that there exists some $\eta>0$ and a constant $\sigma^{2}>0$ such that, as $n \rightarrow \infty$,

$$
\mathbf{E}\left(X_{1}^{(n)}\right)=o\left(\frac{a_{n}}{n}\right), \quad \sup _{n \geq 1} \mathbf{E}\left(\left|X_{1}^{(n)}\right|^{2+\eta}\right)<\infty, \quad \operatorname{Var}\left(X_{1}^{(n)}\right) \rightarrow \sigma^{2} .
$$

The following estimate is essentially due to Mogulskii [31]:

Proposition 2.1 (A triangular version of Mogulskii [31]) Assume [2.4). Let $g_{1}<g_{2}$ be continuous functions on $[0,1]$ with $g_{1}(0)<0<g_{2}(0)$. Consider the measurable event

$$
F_{n}:=\left\{g_{1}\left(\frac{i}{n}\right) \leq \frac{S_{i}^{(n)}}{a_{n}} \leq g_{2}\left(\frac{i}{n}\right), \text { for } 1 \leq i \leq n\right\} .
$$

We have

$$
\lim _{n \rightarrow \infty} \frac{a_{n}^{2}}{n} \log \mathbf{P}\left(F_{n}\right)=-\frac{\pi^{2} \sigma^{2}}{2} \int_{0}^{1} \frac{\mathrm{d} t}{\left[g_{2}(t)-g_{1}(t)\right]^{2}} .
$$

Moreover, for any $b>0$,

$$
\lim _{n \rightarrow \infty} \frac{a_{n}^{2}}{n} \log \mathbf{P}\left\{F_{n}, \frac{S_{n}^{(n)}}{a_{n}} \geq g_{2}(1)-b\right\}=-\frac{\pi^{2} \sigma^{2}}{2} \int_{0}^{1} \frac{\mathrm{d} t}{\left[g_{2}(t)-g_{1}(t)\right]^{2}} .
$$

If the law of $X_{1}^{(n)}$ does not depend on $n$ (in which case we can even take $\eta=0$ ), Proposition 2.1] is Mogulskii [31]'s theorem. For a detailed proof of Proposition 2.1, see [13].

A useful consequence of Proposition 2.1 is as follows. Again, if the law of $X_{1}^{(n)}$ does not depend on $n$, we only need $X_{1}^{(n)}$ to have a finite second moment in order to have (2.4). 
Corollary 2.2 Assume that (2.4) is satisfied with $a_{n}:=n^{1 / 3}$.

(i) Let $f:[0,1] \rightarrow(0, \infty)$ be a continuous function, and let $\left(f_{n}\right)$ be a sequence of continuous functions converging uniformly to $f$ on $[0,1]$. Then for any $b>0$, when $n \rightarrow \infty$,

$$
\sup _{0 \leq u \leq b n^{1 / 3}} \mathbf{P}\left(u \geq S_{i}^{(n)} \geq u-n^{1 / 3} f_{n}\left(\frac{i}{n}\right), 1 \leq i \leq n\right)=\mathrm{e}^{-\frac{\pi^{2} \sigma^{2}}{2}(1+o(1)) n^{1 / 3} \int_{0}^{1} \frac{\mathrm{d} t}{f^{2}(t)}} .
$$

(ii) For any $b>a>0$, we have, as $n \rightarrow \infty$,

$\sum_{j=1}^{n} \mathrm{e}^{-b(n-j)^{1 / 3}} \mathbf{P}\left(a n^{1 / 3} \geq S_{i}^{(n)}>a n^{1 / 3}-b(n-i)^{1 / 3}, \forall 1 \leq i \leq j\right)=\mathrm{e}^{-\min \left\{b, \frac{3 \pi^{2} \sigma^{2}}{2 b^{2}}\right\}(1+o(1)) n^{1 / 3}}$.

Proof of Corollary 2.2. We first prove (ii). Let $\varepsilon>0$. Define $k:=\left\lfloor\frac{1}{\varepsilon}\right\rfloor, n_{\ell}:=\ell\lfloor\varepsilon n\rfloor$ for $\ell=0$, $\cdots, k-1$ and $n_{k}:=n$. By (2.5), the sum in (ii) is, for all large $n$ and some constant $c$,

$$
\begin{aligned}
& \leq\lfloor\varepsilon n\rfloor \sum_{\ell=1}^{k} \mathrm{e}^{-b\left(n-n_{\ell}\right)^{1 / 3}} \mathbf{P}\left(a n^{1 / 3} \geq S_{i}^{(n)}>a n^{1 / 3}-b(n-i)^{1 / 3}, \forall 1 \leq i \leq n_{\ell-1}\right) \\
& \leq\lfloor\varepsilon n\rfloor \sum_{\ell=1}^{k} \mathrm{e}^{-b\left(n-n_{\ell}\right)^{1 / 3}} \mathrm{e}^{-\left(\frac{3 \pi^{2} \sigma^{2}}{2 b^{2}}-\varepsilon\right)\left(n^{1 / 3}-\left(n-n_{\ell-1}\right)^{1 / 3}\right)} \\
& \leq \mathrm{e}^{-\min \left\{b, \frac{3 \pi^{2} \sigma^{2}}{2 b^{2}}\right\}(1-c \varepsilon) n^{1 / 3}} .
\end{aligned}
$$

This proves the upper bound in (ii) as $\varepsilon$ can be arbitrarily small. The lower bound is easier: we only need to consider two terms: $j=\lfloor\varepsilon n\rfloor$ and $j=n$, and apply again (2.5).

The proof of (i) goes along similar lines by cutting the interval $\left\{0 \leq u \leq b n^{1 / 3}\right\}$ into smaller intervals of length of order $\varepsilon n$ with small $\varepsilon>0$, using monotonicity and applying Proposition 2.1. The details are omitted.

We now proceed to the proof of Theorem 1.4 if $\inf _{t \in[0,1]} \mathbf{E}\left\{\sum_{|x|=1} \mathrm{e}^{-t V(x)}\right\}=1$ and $\mathbf{E}\left\{\sum_{|x|=1} V(x) \mathrm{e}^{-V(x)}\right\} \leq 0$, then on the set of non-extinction,

$$
\lim _{n \rightarrow \infty} \frac{1}{n^{1 / 3}} \min _{|x|=n} \bar{V}(x)=\left(\frac{3 \pi^{2} \sigma_{\theta}^{2}}{2}\right)^{1 / 3}, \quad \text { P-a.s. },
$$

where $\sigma_{\theta}^{2}:=\frac{1}{\theta} \mathbf{E}\left\{\sum_{|x|=1} V(x)^{2} \mathrm{e}^{-\theta V(x)}\right\}$ and $\theta \in(0,1]$ is such that $\mathbf{E}\left\{\sum_{|x|=1} V(x) \mathrm{e}^{-\theta V(x)}\right\}=0$.

Without loss of generality, we can assume $\theta=1$. Indeed, if $0<\theta<1$, then by considering $\widetilde{V}(x):=\theta V(x)$, we have $\inf _{t \in[0,1]} \mathbf{E}\left(\sum_{|x|=1} \mathrm{e}^{-t \widetilde{V}(x)}\right)=1$ and $\mathbf{E}\left(\sum_{|x|=1} \widetilde{V}(x) \mathrm{e}^{-\widetilde{V}(x)}\right)=0$, so that by the case $\theta=1, \frac{1}{n^{1 / 3}} \min _{|x|=n} \max _{y \in \rrbracket \varnothing, x \rrbracket} \widetilde{V}(y) \rightarrow\left(\frac{3 \pi^{2} \widetilde{\sigma}^{2}}{2}\right)^{1 / 3} \mathbf{P}$-almost surely on the set of non-extinction, where $\widetilde{\sigma}^{2}:=\mathbf{E}\left\{\sum_{|x|=1} \widetilde{V}(x)^{2} \mathrm{e}^{-\widetilde{V}(x)}\right\}$. 
So we only need to prove Theorem 1.4 in the case $\theta=1$. In the rest of the section, we assume $\mathbf{E}\left(\sum_{|x|=1} \mathrm{e}^{-V(x)}\right)=1$ and $\mathbf{E}\left(\sum_{|x|=1} V(x) \mathrm{e}^{-V(x)}\right)=0$, and prove that, on the set of non-extinction,

$$
\lim _{n \rightarrow \infty} \frac{1}{n^{1 / 3}} \min _{|x|=n} \bar{V}(x)=\left(\frac{3 \pi^{2} \sigma^{2}}{2}\right)^{1 / 3}, \quad \text { P-a.s. },
$$

with $\sigma^{2}:=\sigma_{1}^{2}=\mathbf{E}\left\{\sum_{|x|=1} V(x)^{2} \mathrm{e}^{-V(x)}\right\}$. For the sake of clarity, we prove the upper and lower bounds in distinct parts.

Proof of 2.7): lower bound. We assume $\mathbf{E}\left(\sum_{|x|=1} \mathrm{e}^{-V(x)}\right)=1$ and $\mathbf{E}\left(\sum_{|x|=1} V(x) \mathrm{e}^{-V(x)}\right)=0$. Let $0<a<\left(\frac{3 \pi^{2} \sigma^{2}}{2}\right)^{1 / 3}$ and $b:=\left(\frac{3 \pi^{2} \sigma^{2}}{2}\right)^{1 / 3}$. Let $n \geq 1$. For all $|x|=n$, let

$$
H_{x}:=\inf \left\{j \in[1, n]: V\left(x_{j}\right) \leq a n^{1 / 3}-b(n-j)^{1 / 3}\right\}, \quad \inf \varnothing:=\infty .
$$

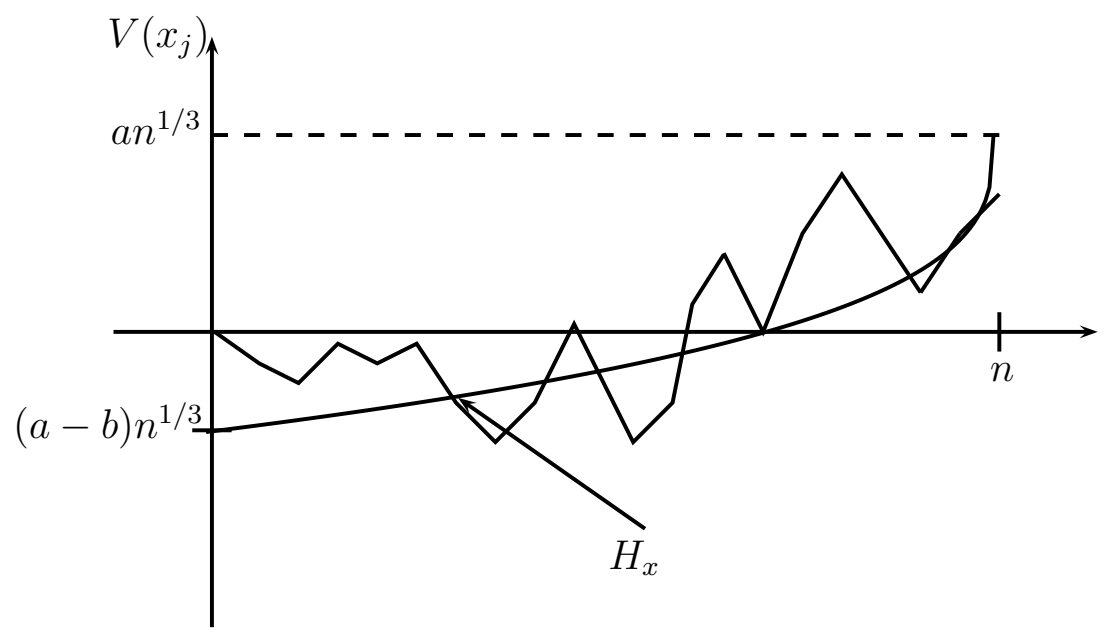

Figure 2: $H_{x}$

Assume there exists a vertex $x$ with $|x|=n$ such that $\bar{V}(x) \leq a n^{1 / 3}$. Then $H_{x} \leq n$; writing $j:=H_{x}$ and $y:=x_{j}$, we have, for all $i<j, a n^{1 / 3} \geq V\left(y_{i}\right)>a n^{1 / 3}-b(n-i)^{1 / 3}$ and $V(y) \leq a n^{1 / 3}-b(n-j)^{1 / 3}$. Therefore, by writing

$$
U_{j}:=\sum_{|y|=j} \mathbf{1}_{\left\{V(y) \leq a n^{1 / 3}-b(n-j)^{1 / 3}, a n^{1 / 3} \geq V\left(y_{i}\right)>a n^{1 / 3}-b(n-i)^{1 / 3}, \forall i<j\right\}},
$$

we obtain:

$$
\mathbf{P}\left(\min _{|x|=n} \bar{V}(x) \leq a n^{1 / 3}\right) \leq \mathbf{P}\left(\bigcup_{j=1}^{n}\left\{U_{j} \geq 1\right\}\right) \leq \sum_{j=1}^{n} \mathbf{E}\left(U_{j}\right) .
$$


By (2.3), we have $\mathbf{E}\left(U_{j}\right)=\mathbf{E}\left[\mathrm{e}^{S_{j}} \mathbf{1}_{\left\{S_{j} \leq a n^{1 / 3}-b(n-j)^{1 / 3}, a n^{1 / 3} \geq S_{i}>a n^{1 / 3}-b(n-i)^{1 / 3}, \forall i<j\right\}}\right]$. Hence

$$
\mathbf{P}\left(\min _{|x|=n} \bar{V}(x) \leq a n^{1 / 3}\right) \leq \sum_{j=1}^{n} \mathrm{e}^{a n^{1 / 3}-b(n-j)^{1 / 3}} \mathbf{P}\left(a n^{1 / 3} \geq S_{i}>a n^{1 / 3}-b(n-i)^{1 / 3}, \forall i<j\right) .
$$

Applying Corollary 2.2 (ii) and noting that $\min \left\{b, \frac{3 \pi^{2} \sigma^{2}}{2 b^{2}}\right\}=\left(\frac{3 \pi^{2} \sigma^{2}}{2}\right)^{1 / 3}$, we get that, for any $0<a<\left(\frac{3 \pi^{2} \sigma^{2}}{2}\right)^{1 / 3}$

$$
\limsup _{n \rightarrow \infty} \frac{1}{n^{1 / 3}} \log \mathbf{P}\left(\min _{|x|=n} \bar{V}(x) \leq a n^{1 / 3}\right) \leq a-\left(\frac{3 \pi^{2} \sigma^{2}}{2}\right)^{1 / 3},
$$

which implies $\sum_{n} \mathbf{P}\left\{\min _{|x|=n} \bar{V}(x) \leq a n^{1 / 3}\right\}<\infty$. The lower bound in (2.7) follows from the Borel-Cantelli lemma, as $a$ can be as close to $\left(\frac{3 \pi^{2} \sigma^{2}}{2}\right)^{1 / 3}$ as possible.

Proof of 2.7): upper bound. Assume $\mathbf{E}\left\{\sum_{|x|=1} \mathrm{e}^{-V(x)}\right\}=1$ and $\mathbf{E}\left\{\sum_{|x|=1} V(x) \mathrm{e}^{-V(x)}\right\}=0$.

Let $n \geq 1$ and $b>a>\varepsilon>0$. The key step in the proof of the upper bound in (2.7) is the following estimate, which is a consequence of the Paley-Zygmund inequality (see [13] for a proof): For any Borel sets $I_{i, n} \subset \mathbb{R}, 1 \leq i \leq n$, and any integer $r_{n} \geq 1$, we have

$$
\mathbf{P}\left\{\exists|x|=n: V\left(x_{i}\right) \in I_{i, n}, \forall 1 \leq i \leq n\right\} \geq \frac{\mathbf{E}\left[\mathrm{e}^{S_{n}} \mathbf{1}_{\left\{S_{i} \in I_{i, n}, \nu_{i-1} \leq r_{n}, \forall 1 \leq i \leq n\right\}}\right]}{1+\left(r_{n}-1\right) \sum_{j=1}^{n} h_{j, n}},
$$

where

$$
h_{j, n}:=\sup _{u \in I_{j, n}} \mathbf{E}\left(\mathrm{e}^{S_{n-j}} \mathbf{1}_{\left\{S_{i} \in I_{i+j, n}-u, \forall 0 \leq i \leq n-j\right\}}\right) \text {, }
$$

and $I_{i+j, n}-u:=\left\{v-u: v \in I_{i+j, n}\right\}$. [We recall that $\left(S_{i}-S_{i-1}, \nu_{i-1}\right), i \geq 1$, are i.i.d. random vectors (with $S_{0}:=0$ ) whose common distribution is given by (2.2) $)$.]

We choose $r_{n}:=\left\lfloor\mathrm{e}^{n^{1 / 4}}\right\rfloor$ and $I_{i, n}:=\left[(a-\varepsilon) n^{1 / 3}-b(n-i)^{1 / 3}, a n^{1 / 3}\right]$. In particular, $\left\{\exists|x|=n: V\left(x_{i}\right) \in I_{i, n}, \forall 1 \leq i \leq n\right\} \subset\left\{\min _{|x|=n} \bar{V}(x) \leq a n^{1 / 3}\right\}$. It follows from (2.9) that

$$
\mathbf{P}\left(\min _{|x|=n} \bar{V}(x) \leq a n^{1 / 3}\right) \geq \frac{\mathrm{e}^{(a-\varepsilon) n^{1 / 3}} \mathbf{P}\left\{S_{i} \in I_{i, n}, \nu_{i-1} \leq \mathrm{e}^{n^{1 / 4}}, \forall 1 \leq i \leq n\right\}}{1+\mathrm{e}^{n^{1 / 4}} \sum_{j=1}^{n} h_{j, n}} .
$$

Let $X_{j}^{(n)}, 1 \leq j \leq n$, be i.i.d. random variables such that $X_{1}^{(n)}$ has the same distribution as $S_{1}$ conditioned on $\left\{\nu_{0} \leq \mathrm{e}^{n^{1 / 4}}\right\}$. Let $S_{0}^{(n)}:=0$ and $S_{j}^{(n)}:=X_{1}^{(n)}+\ldots+X_{j}^{(n)}$ for $1 \leq j \leq n$. Then

$$
\begin{aligned}
& \mathbf{P}\left\{S_{i} \in I_{i, n}, \nu_{i-1} \leq \mathrm{e}^{n^{1 / 4}}, \forall 1 \leq i \leq n\right\} \\
= & {\left[\mathbf{P}\left(\nu_{0} \leq \mathrm{e}^{n^{1 / 4}}\right)\right]^{n} \mathbf{P}\left\{\max _{0 \leq k \leq n} S_{k}^{(n)} \leq a n^{1 / 3}, S_{i}^{(n)} \geq(a-\varepsilon) n^{1 / 3}-b(n-i)^{1 / 3}, \forall 1 \leq i \leq n\right\} . }
\end{aligned}
$$


The second probability expression on the right-hand side is, according to Proposition 2.1 (we easily check that condition (2.4) is satisfied), $=\exp \left\{-(1+o(1)) \frac{\pi^{2} \sigma^{2}}{2} n^{1 / 3} \int_{0}^{1} \frac{\mathrm{d} t}{\left(\varepsilon+b(1-t)^{1 / 3}\right)^{2}}\right\}$, which is bounded by $\exp \left\{-\left(\frac{3 \pi^{2} \sigma^{2}}{2 b^{2}}-c_{1}(\varepsilon)\right) n^{1 / 3}\right\}$ for all sufficiently large $n$, with $c_{1}(\varepsilon)$ denoting a constant such that $\lim _{\varepsilon \rightarrow 0} c_{1}(\varepsilon)=0$. On the other hand, by (2.2), we have, for any $\eta>0$, $\mathbf{E}\left[\left(\nu_{0}\right)^{\eta}\right]=\mathbf{E}\left(N^{\eta} \sum_{i=1}^{N} A_{i}\right)$, which is finite (by Hölder's inequality and (1.2) $)$ if $\eta>0$ is chosen to be sufficiently small; thus $\left[\mathbf{P}\left(\nu_{0} \leq \mathrm{e}^{n^{1 / 4}}\right)\right]^{n} \rightarrow 1$ as $n \rightarrow \infty$. Accordingly, for all sufficiently large $n$ and some constant $c_{2}(\varepsilon)$ satisfying $\lim _{\varepsilon \rightarrow 0} c_{2}(\varepsilon)=0$,

$$
\mathbf{P}\left(\min _{|x|=n} \bar{V}(x) \leq a n^{1 / 3}\right) \geq \frac{\exp \left\{n^{1 / 3}\left[a-\frac{3 \pi^{2} \sigma^{2}}{2 b^{2}}-c_{2}(\varepsilon)\right]\right\}}{1+\mathrm{e}^{n^{1 / 4}} \sum_{j=1}^{n} h_{j, n}}
$$

We now estimate $\sum_{j=1}^{n} h_{j, n}$. By definition,

$$
\begin{aligned}
h_{j, n} & =\sup _{0 \leq u \leq \varepsilon n^{1 / 3}+b(n-j)^{1 / 3}} \mathbf{E}\left(\mathrm{e}^{S_{n-j}} \mathbf{1}_{\left\{u \geq S_{i} \geq u-\varepsilon n^{1 / 3}-b(n-j-i)^{1 / 3}, \forall i \leq n-j\right\}}\right) \\
& \leq \sup _{0 \leq u \leq \varepsilon n^{1 / 3}+b(n-j)^{1 / 3}} \mathrm{e}^{u} \mathbf{P}\left(u \geq S_{i} \geq u-\varepsilon n^{1 / 3}-b(n-j-i)^{1 / 3}, \forall i \leq n-j\right) .
\end{aligned}
$$

Let $A$ be an integer such that $A \geq \frac{1}{\varepsilon^{2}}$. Let $n_{\ell}:=\ell\left\lfloor\frac{n}{A}\right\rfloor$ for $\ell=0,1, \cdots, A-1$ and $n_{A}:=n$. If $j \in\left[n_{\ell}, n_{\ell+1}\right] \cap \mathbb{Z}$ (for some $0 \leq \ell \leq A-1$ ), then

$$
h_{j, n} \leq \mathrm{e}^{\varepsilon n^{1 / 3}+b\left(n-n_{\ell}\right)^{1 / 3}} \sup _{0 \leq u \leq(b+\varepsilon) n^{1 / 3}} \mathbf{P}\left(u \geq S_{i} \geq u-\varepsilon n^{1 / 3}-b\left(n-n_{\ell}-i\right)^{1 / 3}, \forall i \leq n-n_{\ell+1}\right) .
$$

We now bound the supremum on the right-hand side. If $\ell$ is such that $1-\frac{\ell+1}{A} \leq \varepsilon$, then we simply say that the supremum is bounded by 1 , so that $\max _{n_{\ell} \leq j \leq n_{\ell+1}} h_{j, n} \leq \mathrm{e}^{\varepsilon n^{1 / 3}+b\left(n-n_{\ell}\right)^{1 / 3}}$. If $1-\frac{\ell+1}{A}>\varepsilon$, we bound the supremum by applying Corollary 2.2 (i) to $f(t):=\frac{\varepsilon}{\left(1-\frac{\ell+1}{A}\right)^{1 / 3}}+$ $b\left(\frac{A-\ell}{A-(\ell+1)}-t\right)^{1 / 3}:$ since $f(t) \leq \varepsilon^{2 / 3}+b\left(1+\frac{1}{\varepsilon A}-t\right)^{1 / 3} \leq \varepsilon^{2 / 3}+b(1+\varepsilon-t)^{1 / 3}$ (using $A \geq \frac{1}{\varepsilon^{2}}$ for the second inequality), we have $\int_{0}^{1} \frac{\mathrm{d} t}{f^{2}(t)} \geq \frac{3}{b^{2}}-c_{3}(\varepsilon)$, with $c_{3}(\varepsilon)$ denoting a constant satisfying $\lim _{\varepsilon \rightarrow 0} c_{3}(\varepsilon)=0$; hence by Corollary 2.2 (i),

$$
\max _{n_{\ell} \leq j \leq n_{\ell+1}} h_{j, n} \leq \mathrm{e}^{\varepsilon n^{1 / 3}+b\left(n-n_{\ell}\right)^{1 / 3}-\left(\frac{3 \pi^{2} \sigma^{2}}{2 b^{2}}-c_{3}(\varepsilon)\right)\left(n-n_{\ell+1}\right)^{1 / 3}}
$$

Therefore, for all sufficiently large $n$ and a constant $c(\varepsilon)$ satisfying $\lim _{\varepsilon \rightarrow 0} c(\varepsilon)=0$, we have, uniformly in all $\ell \in[0, A-1] \cap \mathbb{Z}$,

$$
\max _{n_{\ell} \leq j \leq n_{\ell+1}} h_{j, n} \leq \mathrm{e}^{n^{1 / 3}\left[\left(b-\frac{3 \pi^{2} \sigma^{2}}{2 b^{2}}\right)^{+}+c(\varepsilon)\right]}
$$


where $u^{+}:=\max \{u, 0\}$. As a consequence, $\max _{0 \leq j \leq n} h_{j, n}=\max _{0 \leq \ell \leq A-1} \max _{n_{\ell} \leq j \leq n_{\ell+1}} h_{j, n} \leq$ $\mathrm{e}^{n^{1 / 3}\left[\left(b-\frac{3 \pi^{2} \sigma^{2}}{2 b^{2}}\right)^{+}+c(\varepsilon)\right]}$ for all sufficiently large $n$. In view of (2.10), we obtain that, for any $b>a>0$,

$$
\liminf _{n \rightarrow \infty} \frac{1}{n^{1 / 3}} \log \mathbf{P}\left(\min _{|x|=n} \bar{V}(x) \leq a n^{1 / 3}\right) \geq-\left(b-\frac{3 \pi^{2} \sigma^{2}}{2 b^{2}}\right)^{+}+a-\frac{3 \pi^{2} \sigma^{2}}{2 b^{2}} .
$$

We now fix $a>\left(\frac{3 \pi^{2} \sigma^{2}}{2}\right)^{1 / 3}$ and $\eta>0$. We can choose $b>a$ sufficiently close to $a$ such that $\left(b-\frac{3 \pi^{2} \sigma^{2}}{2 b^{2}}\right)^{+}-a+\frac{3 \pi^{2} \sigma^{2}}{2 b^{2}}<\eta$; accordingly, for all sufficiently large $n$,

$$
\mathbf{P}\left(\min _{|x|=n} \bar{V}(x) \leq a n^{1 / 3}\right) \geq \mathrm{e}^{-\eta n^{1 / 3}} .
$$

From here, it is routine (McDiarmid [29]) to obtain the upper bound in (2.7); we produce the details for the sake of completeness. Let $R_{n}:=\inf \left\{k: \#\{x:|x|=k\} \geq \mathrm{e}^{2 \eta n^{1 / 3}}\right\}$. For all large $n$,

$$
\begin{aligned}
& \mathbf{P}\left\{R_{n}<\infty, \max _{k \in\left[\frac{n}{2}, n\right]|x|=k+R_{n}} \min _{\leq} \bar{V}(x)>\max _{|y|=R_{n}} \bar{V}(y)+a n^{1 / 3}\right\} \\
\leq & \sum_{k \in\left[\frac{n}{2}, n\right]} \mathbf{P}\left\{R_{n}<\infty, \min _{|x|=k+R_{n}} \bar{V}(x)>\max _{|y|=R_{n}} \bar{V}(y)+a n^{1 / 3}\right\} \\
\leq & \sum_{k \in\left[\frac{n}{2}, n\right]}\left[\mathbf{P}\left\{\min _{|x|=k} \bar{V}(x)>a n^{1 / 3}\right\}\right]^{\left.\mid \mathrm{e}^{2 \eta n^{1 / 3}}\right\rfloor},
\end{aligned}
$$

which, according to (2.12), is summable in $n$. By the Borel-Cantelli lemma, $\mathbf{P}$-a.s. for all large $n$, we have either $R_{n}=\infty$, or $\max _{k \in\left[\frac{n}{2}, n\right]} \min _{|x|=k+R_{n}} \bar{V}(x) \leq \max _{|y|=R_{n}} \bar{V}(y)+a n^{1 / 3}$.

By the law of large numbers for the branching random walk (Biggins [5]), there exists a constant $c \in(0, \infty)$ such that $\frac{1}{n} \max _{|y|=n} V(y) \rightarrow c, \mathbf{P}$-almost surely upon the system's survival. In particular, upon survival, $\max _{|y|=n} V(y) \leq 2 c n, \mathbf{P}$-almost surely for all large $n$. Consequently, upon the system's survival, $\mathbf{P}$-almost surely for all large $n$, we have either $R_{n}=\infty$, or $\max _{k \in\left[\frac{n}{2}, n\right]} \min _{|x|=k+R_{n}} \bar{V}(x) \leq 2 c R_{n}+a n^{1 / 3}$.

Recall that the number of particles in each generation forms a supercritical GaltonWatson process. In particular, conditionally on the system's survival, $\frac{\#\{u:|u|=k\}}{(\mathbf{E} N)^{k}}$ converges a.s. to a (strictly) positive random variable when $k \rightarrow \infty$, which implies $R_{n} \sim 2 \eta \frac{n^{1 / 3}}{\log (\mathbf{E} N)}$ P-a.s. $(n \rightarrow \infty)$, and $\max _{k \in\left[\frac{n}{2}, n\right]} \min _{|x|=k+R_{n}} \bar{V}(x) \geq \min _{|x|=n} \bar{V}(x) \mathbf{P}$-almost surely for all large $n$. As a consequence, upon the system's survival, we have, $\mathbf{P}$-almost surely for all large $n$,

$$
\min _{|x|=n} \bar{V}(x) \leq \frac{5 c \eta}{\log (\mathbf{E} N)} n^{1 / 3}+a n^{1 / 3} .
$$


Since $a$ (resp. $\eta$ ) can be as close to $\left(\frac{3 \pi^{2} \sigma^{2}}{2}\right)^{1 / 3}$ (resp. 0) as possible, this yields the upper bound in (2.7), and completes the proof of Theorem 1.4.

Our proof of Theorem 1.4 gives the following deviation probability of $\min _{|x|=n} \bar{V}(x)$, which may be of independent interest.

Proposition 2.3 Assume $\psi(1)=\psi^{\prime}(1)=0$. For any $0<a \leq\left(\frac{3 \pi^{2} \sigma^{2}}{2}\right)^{1 / 3}$, we have

$$
\lim _{n \rightarrow \infty} \frac{1}{n^{1 / 3}} \log \mathbf{P}\left(\min _{|x|=n} \bar{V}(x) \leq a n^{1 / 3}\right)=a-\left(\frac{3 \pi^{2} \sigma^{2}}{2}\right)^{1 / 3} .
$$

Proof. If $0<a<\left(\frac{3 \pi^{2} \sigma^{2}}{2}\right)^{1 / 3}$, the upper and lower bounds in (2.13) follow from (2.8) and (2.11), respectively. [In (2.11), we use the fact that $b:=\left(\frac{3 \pi^{2} \sigma^{2}}{2}\right)^{1 / 3}$ solves $b=\frac{3 \pi^{2} \sigma^{2}}{2 b^{2}}$.]

If $a=\left(\frac{3 \pi^{2} \sigma^{2}}{2}\right)^{1 / 3}$, only the lower bound in (2.13) requires a proof, which follows immediately from (2.11).

Remark 2.4 Assume $\psi(1)=\psi^{\prime}(1)=0$. Theorem 1.4 says that, on the set of nonextinction, P-almost surely for $n \rightarrow \infty$, there exists $x_{n}$ with $\left|x_{n}\right|=n$ such that $\bar{V}\left(x_{n}\right)=$ $(1+o(1))\left(\frac{3 \pi^{2} \sigma^{2}}{2}\right)^{1 / 3} n^{1 / 3}$. One may wonder whether the vertices $\left(x_{n}\right)$ can be chosen to form an infinite ray (i.e., each $x_{n}$ is a child of $x_{n-1}$ ). The answer is no: Jaffuel [20] proves that this is possible only if we increase the function $\left(\frac{3 \pi^{2} \sigma^{2}}{2}\right)^{1 / 3} n^{1 / 3}$ to $\left(\frac{81 \pi^{2} \sigma^{2}}{8}\right)^{1 / 3} n^{1 / 3}$.

\section{An estimate for one-dimensional random walks}

We present in this section a probability estimate for one-dimensional random walks. It will be used in the proofs of Theorems 1.1 and 1.2 in the forthcoming sections. For each $n \geq 1$, let $X_{i}^{(n)}, 1 \leq i \leq n$, be i.i.d. real-valued variables; let $S_{0}^{(n)}:=0$ and $S_{j}^{(n)}:=\sum_{i=1}^{j} X_{i}^{(n)}$ for $1 \leq j \leq n$. Let $\left(a_{n}\right)$ be positive numbers such that $a_{n} \rightarrow \infty$ and $\frac{a_{n}^{2}}{n} \rightarrow 0, n \rightarrow \infty$. We write $\bar{S}_{j}^{(n)}:=\max _{1 \leq i \leq j} S_{i}^{(n)}$ for $1 \leq j \leq n$.

Proposition 3.1 Assume (2.4). Let $f:[0,1] \rightarrow(0, \infty)$ be a continuous function. For $\delta \geq 0$, we consider the event

$$
G_{\delta}(n):=\left\{(1+\delta) \bar{S}_{j}^{(n)}-S_{j}^{(n)} \leq a_{n} f\left(\frac{j}{n}\right), \forall 1 \leq j \leq n\right\} .
$$

(i) If $\delta=0$, then

$$
\lim _{n \rightarrow \infty} \frac{a_{n}^{2}}{n} \log \mathbf{P}\left\{G_{0}(n)\right\}=-\frac{\pi^{2} \sigma^{2}}{8} \int_{0}^{1} \frac{\mathrm{d} s}{f^{2}(s)}
$$


Moreover, for any fixed $0<b<1$,

$$
\lim _{n \rightarrow \infty} \frac{a_{n}^{2}}{n} \log \mathbf{P}\left\{G_{0}(n), \bar{S}_{n}^{(n)}-S_{n}^{(n)} \leq b a_{n} f(1)\right\}=-\frac{\pi^{2} \sigma^{2}}{8} \int_{0}^{1} \frac{\mathrm{d} s}{f^{2}(s)} .
$$

(ii) If $\delta>0$, then

$$
\lim _{n \rightarrow \infty} \frac{a_{n}^{2}}{n} \log \mathbf{P}\left\{G_{\delta}(n)\right\}=-\frac{\pi^{2} \sigma^{2}}{2} \int_{0}^{1} \frac{\mathrm{d} s}{f^{2}(s)} .
$$

We mention that for the centered random walk $\left(S_{n}\right)$ given in (2.3), assumption (2.4) is obviously satisfied. Hence Proposition 2.1 as well as Corollary 3.2 below, hold also for $\left(S_{n}\right)$.

Proof of Proposition 3.1. (i) Let $0<\varepsilon<\frac{1}{4} \min \left\{b, \min _{0 \leq t \leq 1} f(t)\right\}$ and let $A$ be a large integer. Consider a sufficiently large $n$ such that $\sup _{0 \leq s<t \leq 1, t-s \leq 2 A a_{n}^{2} / n}|f(t)-f(s)| \leq \varepsilon$. Let $m=\left\lfloor\frac{n}{A^{2} a_{n}^{2}}\right\rfloor$. For $0 \leq k<A m$, let $r_{k}:=k\left\lfloor A a_{n}^{2}\right\rfloor$ and $r_{A m}:=n$. Note that $\left\lfloor A a_{n}^{2}\right\rfloor \leq$ $r_{A m}-r_{A m-1} \leq 2\left\lfloor A a_{n}^{2}\right\rfloor$. Let $\ell \in[0, A-1] \cap \mathbb{Z}$ and $k \in[\ell m,(\ell+1) m-1] \cap \mathbb{Z}$. For all $r_{k} \leq j<r_{k+1},\left|f\left(\frac{j}{n}\right)-f\left(\frac{\ell}{A}\right)\right| \leq \varepsilon$. Define

$$
E_{n}^{( \pm)}:=\bigcap_{\ell=0}^{A-1} \bigcap_{k=\ell m}^{(\ell+1) m-1} \bigcap_{j=r_{k}}^{r_{k+1}-1}\left\{\bar{S}_{j}^{(n)}-S_{j}^{(n)} \leq a_{n}\left(f\left(\frac{\ell}{A}\right) \pm \varepsilon\right)\right\} .
$$

Then

$$
\begin{aligned}
\mathbf{P}\left(G_{0}(n)\right) & \leq \mathbf{P}\left(E_{n}^{(+)}\right), \\
\mathbf{P}\left(G_{0}(n), \bar{S}_{n}^{(n)}-S_{n}^{(n)} \leq b a_{n} f\left(\frac{j}{n}\right)\right) & \geq \mathbf{P}\left(E_{n}^{(-)} \cap \bigcap_{0 \leq k \leq A m}\left\{\bar{S}_{r_{k}}^{(n)}-S_{r_{k}}^{(n)} \leq \varepsilon a_{n}\right\}\right) .
\end{aligned}
$$

Observe that for any $r_{k}$, conditionally on $\sigma\left\{S_{j}^{(n)}, 0 \leq j \leq r_{k}\right\}$ and on $\left\{\bar{S}_{r_{k}}^{(n)}-S_{r_{k}}^{(n)}=x\right\}$, the reflecting process $\left(\bar{S}_{i+r_{k}}^{(n)}-S_{i+r_{k}}^{(n)}, 0 \leq i \leq r_{k+1}-r_{k}\right)$ has the same law as $\left(\max \left\{x, \bar{S}_{i}^{(n)}\right\}-\right.$ $\left.S_{i}^{(n)}, 0 \leq i \leq r_{k+1}-r_{k}\right)$. Using this observation for all $k$, we see that

$$
\begin{aligned}
& \mathbf{P}\left(E_{n}^{(+)}\right) \leq \prod_{\ell=0}^{A-1} \prod_{k=\ell m}^{(\ell+1) m-1} \mathbf{P}\left\{\max _{0 \leq i<r_{k+1}-r_{k}}\left(\bar{S}_{i}^{(n)}-S_{i}^{(n)}\right) \leq a_{n}\left(f\left(\frac{\ell}{A}\right)+\varepsilon\right)\right\}, \\
& \mathbf{P}\left(E_{n}^{(-)} \cap \bigcap_{0 \leq k \leq A m}\left\{\bar{S}_{r_{k}}^{(n)}-S_{r_{k}}^{(n)} \leq \varepsilon a_{n}\right\}\right) \geq \prod_{\ell=0}^{A-1} \prod_{k=\ell m}^{(\ell+1) m-1} \mathbf{P}\left\{\Upsilon_{k}\right\},
\end{aligned}
$$

with

$$
\Upsilon_{k}:=\left\{\max _{0 \leq i<r_{k+1}-r_{k}}\left(\bar{S}_{i}^{(n)}-S_{i}^{(n)}\right) \leq a_{n}\left(f\left(\frac{\ell}{A}\right)-2 \varepsilon\right), \bar{S}_{r_{k+1}-r_{k}}^{(n)}-S_{r_{k+1}-r_{k}}^{(n)}<\varepsilon a_{n}, \bar{S}_{r_{k+1}-r_{k}}^{(n)}>\varepsilon a_{n}\right\} .
$$


Now, we prove the upper bound in (i). By (3.1),

$$
\frac{a_{n}^{2}}{n} \log \mathbf{P}\left(E_{n}^{(+)}\right) \leq \frac{m a_{n}^{2}}{n} \sum_{\ell=0}^{A-1} \log \mathbf{P}\left\{\bar{S}_{i}^{(n)}-S_{i}^{(n)} \leq a_{n}\left(f\left(\frac{\ell}{A}\right)+\varepsilon\right), \forall 0 \leq i<\left\lfloor A a_{n}^{2}\right\rfloor\right\} .
$$

According to Donsker's invariance principle 11 the probability term on the right-hand side converges, when $n \rightarrow \infty$, to

$$
\mathbf{P}\left\{\sup _{0 \leq t \leq 1}(\bar{W}(t)-W(t)) \leq \frac{1}{\sigma \sqrt{A}}\left(f\left(\frac{\ell}{A}\right)+\varepsilon\right)\right\},
$$

where $W$ is a standard one-dimensional Brownian motion, and $\bar{W}(t)=\sup _{0 \leq s \leq t} W(s)$. By Lévy's identity, $(\bar{W}(t)-W(t), t \geq 0)$ is distributed as $(|W(t)|, t \geq 0)$; thus we have

$$
\mathbf{P}\left\{\sup _{0 \leq t \leq 1}(\bar{W}(t)-W(t)) \leq u\right\}=\mathrm{e}^{-(1+o(1)) \frac{\pi^{2}}{8 u^{2}}}, \quad u \rightarrow 0,
$$

which can be easily deduced from Formula (5.9) of page 342 of Feller [?], taking $a=2 u$, $t=1$ and $x=u$. As a consequence, for all sufficiently large $A$, say $A \geq A_{0}=A_{0}(\varepsilon, \sigma, f)$,

$$
\log \mathbf{P}\left\{\sup _{0 \leq t \leq 1}(\bar{W}(t)-W(t)) \leq \frac{1}{\sigma \sqrt{A}}\left(f\left(\frac{\ell}{A}\right)+\varepsilon\right)\right\} \leq-\frac{(1-\varepsilon) \pi^{2} \sigma^{2} A}{8\left(f\left(\frac{\ell}{A}\right)+\varepsilon\right)^{2}} .
$$

Since $m \sim \frac{n}{a_{n}^{2} A^{2}}$, we get, for $A \geq A_{0}$,

$$
\begin{aligned}
\limsup _{n \rightarrow \infty} \frac{a_{n}^{2}}{n} \log \mathbf{P}\left(E_{n}^{(+)}\right) & \leq \frac{1}{A^{2}} \sum_{\ell=0}^{A-1} \log \mathbf{P}\left\{\sup _{0 \leq t \leq 1}(\bar{W}(t)-W(t)) \leq \frac{1}{\sigma \sqrt{A}}\left(f\left(\frac{\ell}{A}\right)+\varepsilon\right)\right\} \\
& \leq-\frac{\pi^{2} \sigma^{2}}{8} \frac{1-\varepsilon}{A} \sum_{\ell=0}^{A-1} \frac{1}{\left(f\left(\frac{\ell}{A}\right)+\varepsilon\right)^{2}} .
\end{aligned}
$$

Letting $A \rightarrow \infty$ and then $\varepsilon \rightarrow 0$, we get the upper bound in (i):

$$
\limsup _{n \rightarrow \infty} \frac{a_{n}^{2}}{n} \log \mathbf{P}\left\{\bar{S}_{i}^{(n)}-S_{j}^{(n)} \leq a_{n} f\left(\frac{j}{n}\right), \forall 1 \leq j \leq n\right\} \leq-\frac{\pi^{2} \sigma^{2}}{8} \int_{0}^{1} \frac{\mathrm{d} s}{f^{2}(s)} .
$$

To prove the lower bound in (i), we go back to the events $\Upsilon_{k}$ in (3.2). Observe that for each $1 \leq i \leq r_{k+1}-r_{k}$, all the three events in $\Upsilon_{k}$ are non-decreasing with respect to $S_{i}^{(n)}-S_{i-1}^{(n)}$. By the FKG inequality,

$$
\begin{aligned}
\mathbf{P}\left(\Upsilon_{k}\right) \geq \mathbf{P}( & \left.\max _{0 \leq i<r_{k+1}-r_{k}}\left(\bar{S}_{i}^{(n)}-S_{i}^{(n)}\right) \leq a_{n}\left(f\left(\frac{\ell}{A}\right)-2 \varepsilon\right)\right) \\
& \times \mathbf{P}\left(\bar{S}_{r_{k+1}-r_{k}}^{(n)}-S_{r_{k+1}-r_{k}}^{(n)}<\varepsilon a_{n}\right) \mathbf{P}\left(\bar{S}_{r_{k+1}-r_{k}}^{(n)}>\varepsilon a_{n}\right) .
\end{aligned}
$$

\footnotetext{
${ }^{1}$ Finite-dimensional convergence is checked by Lindeberg's condition in the central limit theorem, whereas tightness is proved via a standard argument as in Billingsley [8].
} 
Recall that $r_{k+1}-r_{k}=\left\lfloor A a_{n}^{2}\right\rfloor$ for $0 \leq k<A m-1$, and $\left\lfloor A a_{n}^{2}\right\rfloor \leq r_{A m}-r_{A m-1} \leq 2\left\lfloor A a_{n}^{2}\right\rfloor$. Using Donsker's invariance principle again, we see that there exists a constant $c(\varepsilon)>0$ such that for all $k, \mathbf{P}\left(\bar{S}_{r_{k+1}-r_{k}}^{(n)}-S_{r_{k+1}-r_{k}}^{(n)}<\varepsilon a_{n}\right) \mathbf{P}\left(\bar{S}_{r_{k+1}-r_{k}}^{(n)}>\varepsilon a_{n}\right) \geq c(\varepsilon)$. From this, the lower bound in (i) follows in the same way as the upper bound in (i).

(ii) Let us first prove the following fact: for any fixed $c>0$,

$$
\mathbf{P}\left\{\sup _{0 \leq s \leq 1}(\bar{W}(s)-W(s)) \leq u, \bar{W}(1) \leq c u\right\}=\mathrm{e}^{-\frac{\pi^{2}}{2 u^{2}}(1+o(1))}, \quad u \rightarrow 0 .
$$

To see why (3.4) holds, we denote by $L(t)$ the local time at 0 of $W$ up to time $t$, and recall from Borodin and Salminen ([9], page 259, Formula 1.16.2) that, for $\lambda>0$,

$$
\int_{0}^{\infty} \mathrm{e}^{-\lambda t} \mathbf{P}\left(\sup _{s \leq t}|W(s)| \leq 1, L(t) \leq c\right) \mathrm{d} t=\frac{1}{\lambda}\left(1-\frac{1}{\cosh (\sqrt{2 \lambda})}\right)\left(1-\mathrm{e}^{-c \sqrt{\frac{\lambda}{2}} \operatorname{coth}(\sqrt{2 \lambda})}\right) .
$$

By analytic continuation, we get that for $0<\lambda<\frac{\pi^{2}}{2}$,

$$
\int_{0}^{\infty} \mathrm{e}^{\lambda t} \mathbf{P}\left(\sup _{s \leq t}|W(s)| \leq 1, L(t) \leq c\right) \mathrm{d} t=\frac{1}{\lambda}\left(\frac{1}{\cos (\sqrt{2 \lambda})}-1\right)\left(1-\mathrm{e}^{-c \sqrt{\frac{\lambda}{2}} \operatorname{cotan}(\sqrt{2 \lambda})}\right) .
$$

This implies, by means of a Tauberian theorem (see, for example, Theorem 3.2 of [16]), that

$$
\mathbf{P}\left(\sup _{0 \leq s \leq t}|W(s)| \leq 1, L(t) \leq c\right)=\mathrm{e}^{-\left(\frac{\pi^{2}}{2}+o(1)\right) t}, \quad t \rightarrow \infty
$$

which, by scaling, is equivalent to $\mathbf{P}\left(\sup _{0 \leq s \leq 1}|W(s)| \leq u, L(1) \leq \frac{u}{\delta}\right)=\mathrm{e}^{-\left(\frac{\pi^{2}}{2 u}+o(1)\right)}, u \rightarrow 0$. By Lévy's identity, the two processes $(\bar{W}-W, \bar{W})$ and $(|W|, L)$ have the same law; consequently, this implies (3.4).

Now let us proceed to prove the upper bound in (ii). Let $\varepsilon>0$, and let $\left(r_{k}\right)$ be as in the proof of (i), i.e., $A$ is a large integer, $m:=\left\lfloor\frac{n}{A^{2} a_{n}^{2}}\right\rfloor, r_{k}:=k\left\lfloor A a_{n}^{2}\right\rfloor$ (for $0 \leq k<A m$ ) and $r_{A m}:=n$, with $n$ sufficiently large such that $\left|f\left(\frac{j}{n}\right)-f\left(\frac{\ell}{A}\right)\right| \leq \varepsilon$ for $r_{k} \leq j \leq r_{k+1}$ and $k \in[\ell m,(\ell+1) m) \cap \mathbb{Z}$. Let

$$
F_{n}^{(+)}:=\bigcap_{\ell=0}^{A-1} \bigcap_{k=\ell m}^{(\ell+1) m-1}\left\{\max _{r_{k} \leq j \leq r_{k+1}}\left(\bar{S}_{j}^{(n)}-S_{j}^{(n)}\right) \leq a_{n}\left(f\left(\frac{\ell}{A}\right)+\varepsilon\right), \bar{S}_{r_{k+1}}^{(n)} \leq \frac{c}{\delta} a_{n}\right\}
$$

where $c=\sup _{0 \leq t \leq 1} f(t)$. Clearly $G_{\delta}(n) \subset F_{n}^{(+)}$. The Markov property yields that for each $k$, conditionally on $\sigma\left\{S_{j}^{(n)}, 0 \leq j \leq r_{k}\right\}$ and on $\left\{\bar{S}_{r_{k}}^{(n)}-S_{r_{k}}^{(n)}=x_{k}, S_{r_{k}}^{(n)}=y_{k}\right\}$, the process $\left(\bar{S}_{i+r_{k}}^{(n)}, \bar{S}_{i+r_{k}}^{(n)}-S_{i+r_{k}}^{(n)}, 0 \leq i \leq r_{k+1}-r_{k}\right)$ has the same law as $\left(\max \left\{x_{k}, \bar{S}_{i}^{(n)}\right\}+\right.$ 
$\left.y_{k}, \max \left\{x_{k}, \bar{S}_{i}^{(n)}\right\}-S_{i}^{(n)}, 0 \leq i \leq r_{k+1}-r_{k}\right)$. On $F_{n}^{(+)}$, we have $-y_{k} \leq x_{k} \leq(c+\varepsilon) a_{n}$ (recalling that $c=\sup _{0 \leq t \leq 1} f(t)$ ), thus $y_{k} \geq-(c+\varepsilon) a_{n}$. Therefore, by the Markov property,

$\mathbf{P}\left(F_{n}^{(+)}\right) \leq \prod_{\ell=0}^{A-1} \prod_{k=\ell m}^{(\ell+1) m-1} \mathbf{P}\left\{\max _{0 \leq i \leq r_{k+1}-r_{k}}\left(\bar{S}_{i}^{(n)}-S_{i}^{(n)}\right) \leq a_{n}\left(f\left(\frac{\ell}{A}\right)+\varepsilon\right), \bar{S}_{r_{k+1}-r_{k}}^{(n)} \leq\left(c+\varepsilon+\frac{c}{\delta}\right) a_{n}\right\}$.

This is the analogue of (3.1) for (ii). From here, the rest of the proof of the upper bound in (ii) is done by using exactly the same arguments as in (i), by applying (3.4) instead of (3.3). We omit the details.

The proof of the lower bound in (ii) is easy. Indeed, let $0<\varepsilon<\inf _{t \in[0,1]} f(t)$, and let

$$
F_{n}^{(-)}:=\left\{-\left(f\left(\frac{i}{n}\right)-\varepsilon\right) \leq \frac{S_{i}^{(n)}}{a_{n}} \leq \frac{\varepsilon}{1+\delta}, \forall 0 \leq i \leq n\right\}
$$

Clearly $F_{n}^{(-)} \subset G_{\delta}(n)$. By (2.5) $)$, we have

$$
\lim _{n \rightarrow \infty} \frac{a_{n}^{2}}{n} \log \mathbf{P}\left(F_{n}^{(-)}\right)=-\frac{\pi^{2} \sigma^{2}}{2} \int_{0}^{1} \frac{\mathrm{d} t}{\left(f(t)-\varepsilon+\frac{\varepsilon}{1+\delta}\right)^{2}} .
$$

Letting $\varepsilon \rightarrow 0$ gives the lower bound in (ii).

The following corollary follows from Proposition 3.1 exactly as Corollary 2.2 follows from Proposition 2.1.

Corollary 3.2 Assume that 2.4 is satisfied with $a_{n}=n^{1 / 3}$. Let $a>0$ and $\delta>0$. Then for $n \rightarrow \infty$,

$$
\begin{aligned}
\sum_{j=1}^{n} \mathrm{e}^{-a(n-j)^{1 / 3}} \mathbf{P}\left(\bar{S}_{i}^{(n)}-S_{i}^{(n)} \leq a(n-i)^{1 / 3}, \forall 1 \leq i \leq j\right) & =\mathrm{e}^{-\min \left\{a, \frac{3 \pi^{2} \sigma^{2}}{8 a^{2}}\right\}(1+o(1)) n^{1 / 3}}, \\
\sum_{j=1}^{n} \mathrm{e}^{-a(n-j)^{1 / 3}} \mathbf{P}\left((1+\delta) \bar{S}_{i}^{(n)}-S_{i}^{(n)} \leq a(n-i)^{1 / 3}, \forall 1 \leq i \leq j\right) & =\mathrm{e}^{-\min \left\{a, \frac{3 \pi^{2} \sigma^{2}}{2 a^{2}}\right\}(1+o(1)) n^{1 / 3}} .
\end{aligned}
$$

\section{Proof of Theorem 1.2}

We assume $\inf _{t \in[0,1]} \psi(t)=0$ and $\psi^{\prime}(1) \geq 0$ in this section. Let $\theta \in(0,1]$ be such that $\psi^{\prime}(\theta)=0$ as in (1.4). By Theorem 1.4 and (1.7), we get that, on the set of non-extinction,

$$
\liminf _{n \rightarrow \infty} \frac{1}{n^{1 / 3}} \log \varrho_{n} \geq-\alpha_{\theta}^{1 / 3}, \quad \text { P-a.s. }
$$


where $\alpha_{\theta}:=\frac{3 \pi^{2}}{2 \theta} \mathbf{E}\left[\sum_{i=1}^{N} A_{i}^{\theta}\left(\log A_{i}\right)^{2}\right]=\frac{3 \pi^{2}}{2 \theta} \mathbf{E}\left[\sum_{|x|=1} V(x)^{2} \mathrm{e}^{-\theta V(x)}\right]$, and $\varrho_{n}:=P_{\omega}\left\{\tau_{n}<\tau_{0}\right\}$ is as in (1.7). In view of Fact 1.3, it remains only to check that if $\psi^{\prime}(1)>0$ (i.e., if $\theta<1$ ), then we have, on the set of non-extinction,

$$
\limsup _{n \rightarrow \infty} \frac{1}{n^{1 / 3}} \log \varrho_{n} \leq-\alpha_{\theta}^{1 / 3}, \quad \text { P-a.s. }
$$

We do not assume $\psi^{\prime}(1)>0$ for the moment (so $\theta$ can be 1 , and the inequality (4.2) below can also be used in the proof of Theorem 1.1 in the next section). Let $a>0, n \geq 1$ and $\delta \geq 0$. For any $y$ with $|y| \leq n$, say $|y|=j$, we introduce the following event:

$$
E_{\delta}(y)=\left\{(1+\delta) \bar{V}(y)-V(y) \geq \frac{a}{\theta}(n-j)^{1 / 3}\right\} \cap \bigcap_{i=1}^{j-1}\left\{(1+\delta) \bar{V}\left(y_{i}\right)-V\left(y_{i}\right)<\frac{a}{\theta}(n-i)^{1 / 3}\right\},
$$

where $y_{i}$ is the unique vertex of $\llbracket \varnothing, y \rrbracket$ that is in the $i$-th generation, whereas $\bar{V}(x):=$ $\max _{z \in \rrbracket \varnothing, x \rrbracket} V(z)$ as in (1.8).
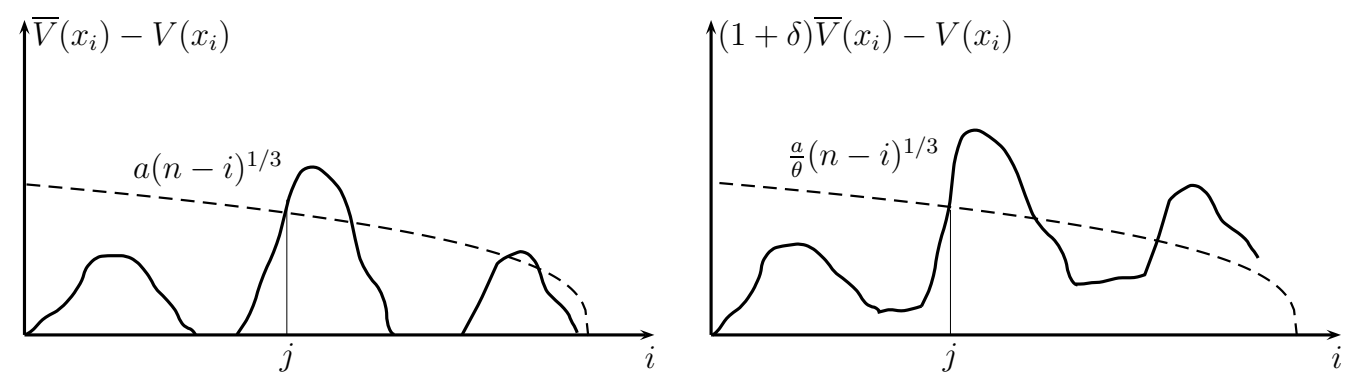

Figure 3: $j=\inf \left\{i: E_{\delta}\left(x_{i}\right)\right.$ holds. $\}$

Let as before $\tau_{n}:=\inf \left\{i \geq 1:\left|X_{i}\right|=n\right\}$ and $T(x):=\inf \left\{k \geq 0: X_{k}=x\right\}$. Consider any vertex $x$ with $|x|=n$. Let $j=j(x) \in[1, n] \cap \mathbb{Z}$ be the smallest integer such that $(1+\delta) \bar{V}\left(x_{j}\right)-V\left(x_{j}\right) \geq \frac{a}{\theta}(n-j)^{1 / 3}$. Such a $j$ exists. Moreover, we have $T(x) \geq T\left(x_{j}\right)$, and $E_{\delta}\left(x_{j}\right)$ holds. Consequently,

$$
\tau_{n}=\inf _{|x|=n} T(x) \geq \min _{1 \leq j \leq n} \inf \left\{T(y):|y|=j \text { and } E_{\delta}(y) \text { holds }\right\},
$$

so that $\varrho_{n}=P_{\omega}\left\{\tau_{n}<\tau_{0}\right\} \leq \sum_{j=1}^{n} \sum_{|y|=j} \mathbf{1}_{E_{\delta}(y)} P_{\omega}\left\{T(y)<\tau_{0}\right\}$. By (1.11), we obtain:

$$
\varrho_{n} \leq \sum_{j=1}^{n} \sum_{|y|=j} \mathbf{1}_{E_{\delta}(y)} \omega\left(\varnothing, y_{1}\right) \mathrm{e}^{V\left(y_{1}\right)-\bar{V}(y)}=\omega(\varnothing, \overleftarrow{\varnothing}) \sum_{j=1}^{n} \sum_{|y|=j} \mathbf{1}_{E_{\delta}(y)} \mathrm{e}^{-\bar{V}(y)}
$$

which is bounded by $\sum_{j=1}^{n} \sum_{|y|=j} \mathbf{1}_{E_{\delta}(y)} \mathrm{e}^{-\bar{V}(y)}$. 
We now assume furthermore $\psi^{\prime}(1)>0$, so that $\theta<1$. We choose $\delta \in\left(0, \frac{1}{\theta}-1\right)$. Since $(1+\delta) \theta<1$, we have

$$
\varrho_{n}^{(1+\delta) \theta} \leq \sum_{j=1}^{n} \sum_{|y|=j} \mathbf{1}_{E_{\delta}(y)} \mathrm{e}^{-(1+\delta) \theta \bar{V}(y)}
$$

Consider the branching random walk $\widetilde{V}(x):=\theta V(x)$ for any $x$. If we define $\widetilde{\psi}(t):=$ $\log \mathbf{E}\left[\sum_{|x|=1} \mathrm{e}^{-t \widetilde{V}(x)}\right]$, then $\widetilde{\psi}(1)=\widetilde{\psi}^{\prime}(1)=0$. We apply formula (2.3) to $(\widetilde{V}(x))$, and obtain a centered one-dimensional random walk $\left(\widetilde{S}_{i}, 0 \leq i \leq n\right)$ with $\widetilde{\sigma}^{2}:=\mathbf{E}\left(\widetilde{S}_{1}^{2}\right)=$ $\mathbf{E}\left[\sum_{|x|=1} \theta^{2} V(x)^{2} \mathrm{e}^{-\theta V(x)}\right]$ such that for $1 \leq j \leq n\left(\right.$ writing $\left.\widehat{S}_{i}:=\max _{1 \leq k \leq i} \widetilde{S}_{k}, \forall i\right)$,

$$
\begin{aligned}
\mathbf{E}\left(\sum_{|y|=j} \mathbf{1}_{E_{\delta}(y)} \mathrm{e}^{-(1+\delta) \theta \bar{V}(y)}\right) & =\mathbf{E}\left(\mathrm{e}^{\widetilde{S}_{j}-(1+\delta) \widehat{S}_{j}} \mathbf{1}_{\left\{(1+\delta) \widehat{S}_{i}-\widetilde{S}_{i}<a(n-i)^{1 / 3}, \forall i<j,(1+\delta) \widehat{S}_{j}-\widetilde{S}_{j} \geq a(n-j)^{1 / 3}\right\}}\right) \\
& \leq \mathrm{e}^{-a(n-j)^{1 / 3}} \mathbf{P}\left((1+\delta) \widehat{S}_{i}-\widetilde{S}_{i}<a(n-i)^{1 / 3}, \forall i<j\right) .
\end{aligned}
$$

It follows that

$$
\mathbf{E}\left(\varrho_{n}^{(1+\delta) \theta}\right) \leq \sum_{j=1}^{n} \mathrm{e}^{-a(n-j)^{1 / 3}} \mathbf{P}\left((1+\delta) \widehat{S}_{i}-\widetilde{S}_{i}<a(n-i)^{1 / 3}, \forall i<j\right) .
$$

We choose $a:=\left(\frac{3 \pi^{2} \widetilde{\sigma}^{2}}{2}\right)^{1 / 3}=\theta \alpha_{\theta}^{1 / 3}$. Applying Corollary 3.2 (ii) to $\left(\widetilde{S}_{i}\right)$, we get $\mathbf{E}\left(\varrho_{n}^{(1+\delta) \theta}\right) \leq$ $\mathrm{e}^{-(a+o(1)) n^{1 / 3}}$, for $n \rightarrow \infty$. By Chebyshev's inequality and the Borel-Cantelli lemma, Palmost surely for $n \rightarrow \infty, \varrho_{n}^{(1+\delta) \theta} \leq \mathrm{e}^{-(a+o(1)) n^{1 / 3}}$. Since $\delta$ can be arbitrarily small, this implies (4.1), and completes the proof of Theorem 1.2 .

\section{Proof of Theorem 1.1: upper bound}

We prove that if $\psi(1)=\psi^{\prime}(1)=0$, then 2

$$
\limsup _{n \rightarrow \infty} \frac{\max _{0 \leq k \leq n}\left|X_{k}\right|}{(\log n)^{3}} \leq \frac{8}{3 \pi^{2} \sigma^{2}}, \quad \text { P-a.s. }
$$

where $\sigma^{2}:=\mathbf{E}\left\{\sum_{|x|=1} V(x)^{2} \mathrm{e}^{-V(x)}\right\}$.

Let, for any $n \geq 1$,

$$
\beta_{n}:=P_{\omega}\left\{\tau_{n}<T_{\overleftarrow{\varnothing}}\right\},
$$

where $\tau_{n}:=\inf \left\{i \geq 1:\left|X_{i}\right|=n\right\}$ is as before the first time that the walk reaches the $n$-th generation, whereas $T_{\overleftarrow{\varnothing}}:=\inf \left\{i \geq 0: X_{i}=\overleftarrow{\varnothing}\right\}$ is the first time that the walk hits $\overleftarrow{\varnothing}$. There

\footnotetext{
${ }^{2}$ On the set of extinction, the upper bound is, in fact, trivially true.
} 
is a simple relation between $\beta_{n}$ and $\varrho_{n}:=P_{\omega}\left\{\tau_{n}<\tau_{0}\right\}$, as stated in the following lemma. We mention that no condition on $\psi$ is in force for the lemma.

Lemma 5.1 Assume that the walk $\left(X_{n}\right)$ is recurrent. We have, for all $n \geq 1$,

$$
\varrho_{n} \leq \beta_{n} \leq \frac{\varrho_{n}}{\omega(\varnothing, \overleftarrow{\varnothing})}
$$

Proof of Lemma 5.1. The first inequality in (5.3) is trivial. Let us prove the second. Let $T_{\varnothing}^{(0)}:=0$ and $T_{\varnothing}^{(k)}:=\inf \left\{i>T_{\varnothing}^{(k-1)}: X_{i}=\varnothing\right\}$ (for $k \geq 1$ ). In words, $T_{\varnothing}^{(k)}$ is the $k$-th return time to the root $\varnothing$. [Thus $T_{\varnothing}^{(1)}=\tau_{0}$.] Since the walk is recurrent, each $T_{\varnothing}^{(k)}$ is well-defined.

Recall that $\beta_{n}$ represents the probability that, starting from the root, the walk visits generation $n$ before hitting $\overleftarrow{\varnothing}$. By considering the number of returns to $\varnothing$ (which can be 0 ) by the walk before visiting generation $n$, we have

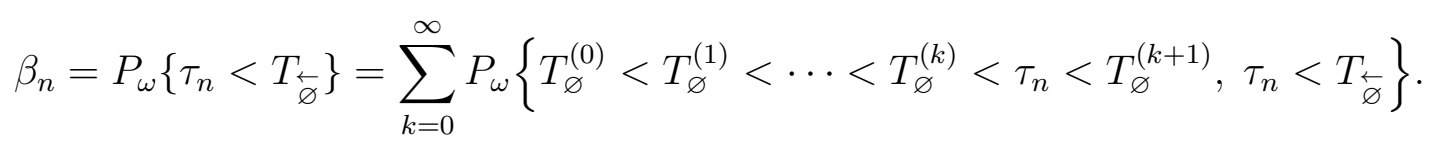

Applying the strong Markov property successively at $T_{\varnothing}^{(k)}, \cdots, T_{\varnothing}^{(1)}$, we see that the prob-

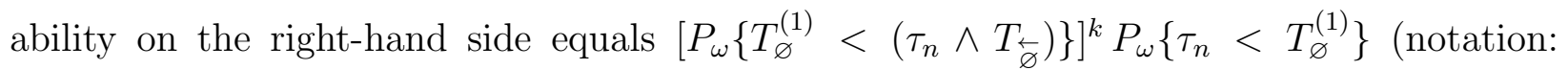
$u \wedge v:=\min \{u, v\})$. Therefore

$$
\beta_{n}=\frac{P_{\omega}\left\{\tau_{n}<T_{\varnothing}^{(1)}\right\}}{1-P_{\omega}\left\{T_{\varnothing}^{(1)}<\left(\tau_{n} \wedge T_{\overleftarrow{\varnothing}}\right)\right\}}=\frac{\varrho_{n}}{1-P_{\omega}\left\{\tau_{0}<\left(\tau_{n} \wedge T_{\overleftarrow{\varnothing}}\right)\right\}} .
$$

Since $1-P_{\omega}\left\{\tau_{0}<\left(\tau_{n} \wedge T_{\overleftarrow{\varnothing}}\right)\right\} \geq 1-P_{\omega}\left\{\tau_{0}<T_{\overleftarrow{\varnothing}}\right\}=\omega(\varnothing, \overleftarrow{\varnothing})$, this yields the lemma.

We now turn to the proof of (5.1). Assume $\psi(1)=\psi^{\prime}(1)=0$. We claim that it suffices to prove that

$$
\limsup _{n \rightarrow \infty} \frac{1}{n^{1 / 3}} \log \mathbf{E}\left(\beta_{n}\right) \leq-\left(\frac{3 \pi^{2} \sigma^{2}}{8}\right)^{1 / 3} .
$$

Indeed, if (5.4) holds, then by Chebyshev's inequality and the Borel-Cantelli lemma, for any $\varepsilon>0$ and $\mathbf{P}$-almost surely all sufficiently large $n, \beta_{n} \leq \exp \left[-(1-\varepsilon)\left(\frac{3 \pi^{2} \sigma^{2}}{8}\right)^{1 / 3} n^{1 / 3}\right]$, which by Lemma 5.1 yields $\varrho_{n} \leq \exp \left[-(1-\varepsilon)\left(\frac{3 \pi^{2} \sigma^{2}}{8}\right)^{1 / 3} n^{1 / 3}\right]$. In view of Fact 1.3. we obtain (5.11).

It remains to prove (5.4). Let $a:=\left(\frac{3 \pi^{2} \sigma^{2}}{8}\right)^{1 / 3}$ and $n \geq 1$. By (4.2) and Lemma 5.1,

$$
\mathbf{E}\left(\beta_{n}\right) \leq \sum_{j=1}^{n} \mathbf{E}\left(\sum_{|y|=j} \mathbf{1}_{E_{0}(y)} \mathrm{e}^{-\bar{V}(y)}\right)
$$


where

$$
E_{0}(y):=\left\{\bar{V}(y)-V(y) \geq a(n-j)^{1 / 3}\right\} \cap \bigcap_{i=1}^{j-1}\left\{\bar{V}\left(y_{i}\right)-V\left(y_{i}\right)<a(n-i)^{1 / 3}\right\} .
$$

Applying (2.3), this leads to (with $\bar{S}_{j}:=\max _{1 \leq i \leq j} S_{i}$ as before):

$$
\begin{aligned}
\mathbf{E}\left(\beta_{n}\right) & \leq \sum_{j=1}^{n} \mathbf{E}\left\{\mathrm{e}^{S_{j}} \mathbf{1}_{\left\{\bar{S}_{j}-S_{j} \geq a(n-j)^{1 / 3}, \bar{S}_{i}-S_{i}<a(n-i)^{1 / 3}, \forall i<j\right\}} \mathrm{e}^{-\bar{S}_{j}}\right\} \\
& \leq \sum_{j=1}^{n} \mathrm{e}^{-a(n-j)^{1 / 3}} \mathbf{P}\left\{\bar{S}_{i}-S_{i}<a(n-i)^{1 / 3}, \forall i<j\right\},
\end{aligned}
$$

which, according to Corollary $3.2(\mathrm{i})$, is bounded by $\exp \left[-(1+o(1))\left(\frac{3 \pi^{2} \sigma^{2}}{8}\right)^{1 / 3} n^{1 / 3}\right]$ for $n \rightarrow \infty$. This yields (5.4).

\section{Proof of Theorem 1.1: lower bound}

We start by recalling a spinal decomposition for the branching random walk $(V(x))$. This decomposition has been used in the literature by many authors in various forms, going back at least to Kahane and Peyrière [21]. The material in this paragraph is borrowed from Lyons, Pemantle and Peres [26] and Lyons [24]. The starting point is to a change-of-probabilities technique on the space of trees; we refer to the aforementioned references for more precision.

Assume $\psi(1)=0$, i.e., $\mathbf{E}\left\{\sum_{|x|=1} \mathrm{e}^{-V(x)}\right\}=1$. Let

$$
W_{n}:=\sum_{|x|=n} \mathrm{e}^{-V(x)}, \quad n \geq 0 .
$$

Clearly, $\left(W_{n}\right)$ is a martingale with respect to the filtration $\left(\mathscr{F}_{n}\right)$, where $\mathscr{F}_{n}$ is the sigmaalgebra generated by the branching random walk in the first $n$ generations.

By Kolmogorov's extension theorem, there exists a probability $\mathbf{Q}$ on $\mathscr{F}_{\infty}$ (the sigmaalgebra generated by the branching random walk) such that for any $n$,

$$
\mathbf{Q}_{\left.\right|_{\mathscr{F}}}=W_{n} \bullet \mathbf{P}_{\mid \mathscr{F} n},
$$

i.e., $\mathbf{Q}(A)=\mathbf{E}\left(W_{n} \mathbf{1}_{A}\right), \forall A \in \mathscr{F}_{n}$. The law of the branching random walk under the new probability $\mathbf{Q}$ is called the law of a size-biased branching random walk. It is clear that the size-biased branching random walk survives with probability one. 
There is a one-to-one correspondence between a branching random walk and a marked tree. On the enlarged probability space formed by marked trees with distinguished rays, we may construct a probability $\mathbf{Q}$ satisfying (6.1), and an infinite ray $\left\{w_{0}=\varnothing, w_{1}, \ldots, w_{n}, ..\right\}$ such that for any $n \geq 1, \overleftarrow{w}_{n}=w_{n-1}$ (recalling that $\overleftarrow{x}$ is the parent of $x$ ) and

$$
\mathbf{Q}\left\{w_{n}=x \mid \mathscr{F}_{n}\right\}=\frac{\mathrm{e}^{-V(x)}}{W_{n}}, \quad \forall|x|=n .
$$

For any individual $x \neq \varnothing$, let

$$
\Delta V(x):=V(x)-V(\overleftarrow{x})
$$

We write, for $k \geq 1$,

$$
\mathscr{I}_{k}:=\left\{x:|x|=k, \overleftarrow{x}=w_{k-1}, x \neq w_{k}\right\}
$$

In words, $\mathscr{I}_{k}$ is the set of children of $w_{k-1}$ except $w_{k}$, or equivalently, the set of the brothers of $w_{k}$, and is possibly empty. Finally, let us introduce the following sigma-field:

$$
\mathscr{G}_{n}:=\sigma\left\{\left(\Delta V(x), x \in \mathscr{I}_{k}\right), V\left(w_{k}\right), w_{k}, \mathscr{I}_{k}, 1 \leq k \leq n\right\} .
$$

The promised spinal decomposition is as follows ( $x u$ denoting concatenation of $x$ and $u$ ). Although it slightly differs from the spinal decomposition presented in Lyons [24], we feel free to omit the proof.

Proposition 6.1 Assume $\psi(1)=0$, and fix $n \geq 1$. Under probability $\mathbf{Q}$,

(i) the random variables $\left(\Delta V\left(w_{k}\right), \Delta V(x), x \in \mathscr{I}_{k}\right), 1 \leq k \leq n$, are i.i.d.;

(ii) conditionally on $\mathscr{G}_{n}$, the shifted branching random walks $\left(\{V(x u)-V(x)\}_{|u|=k}, 0 \leq\right.$ $k \leq n-|x|)$, for $x \in \bigcup_{k=1}^{n} \mathscr{I}_{k}$, are independent, and have the same law as $\left(\{V(u)\}_{|u|=k}, 0 \leq\right.$ $k \leq n-|x|)$ under $\mathbf{P}$.

We now proceed to (the beginning of) the proof of the lower bound in Theorem 1.1, of which we recall the statement: under the assumption $\psi(1)=\psi^{\prime}(1)=0$, we have, on the set of non-extinction,

$$
\liminf _{n \rightarrow \infty} \frac{\max _{0 \leq k \leq n}\left|X_{k}\right|}{(\log n)^{3}} \geq \frac{4}{\alpha}=\frac{8}{3 \pi^{2} \sigma^{2}}, \quad \mathbb{P} \text {-a.s. }
$$

where $\sigma^{2}:=\mathbf{E}\left\{\sum_{|x|=1} V(x)^{2} \mathrm{e}^{-V(x)}\right\}$. 


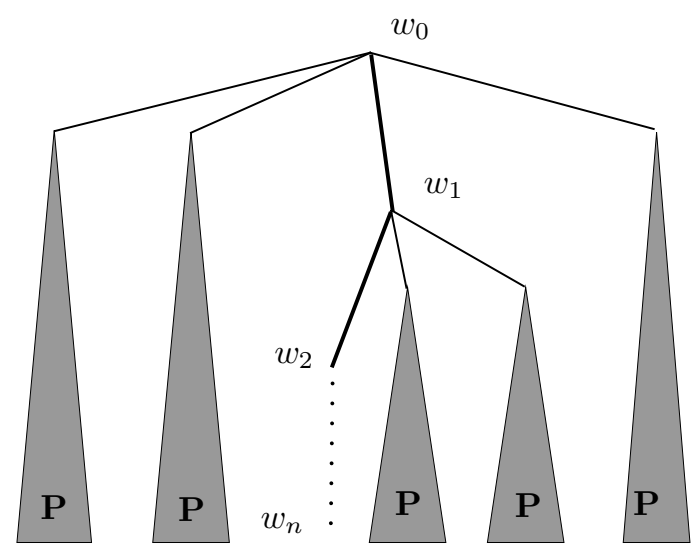

Figure 4: A Q-tree

Let $\beta_{n}:=P_{\omega}\left\{\tau_{n}<T_{\overleftarrow{\varnothing}}\right\}$ be as in (5.2), where $\tau_{n}=\inf \left\{i \geq 1:\left|X_{i}\right|=n\right\}$, and $T_{\overleftarrow{\varnothing}}=$ $\inf \left\{i \geq 0: X_{i}=\overleftarrow{\varnothing}\right\}$. We claim that it suffices to prove that

$$
\liminf _{n \rightarrow \infty} \frac{1}{n^{1 / 3}} \log \mathbf{E}\left(\beta_{n}\right) \geq-\left(\frac{3 \pi^{2} \sigma^{2}}{8}\right)^{1 / 3} .
$$

It is indeed easy to check that (6.6) implies (6.5)): Let $\mathscr{S}:=\{$ the system survives $\}$, $\mathscr{S}_{n}:=\{$ the system survives at least until generation $n\}$. Clearly $\mathscr{S} \subset \mathscr{S}_{n}$ for any $n$. Recall that there exists (see [19], p. 755) a constant $c>0$ such that for all large $n$,

$$
\mathbf{P}\left(W_{n}<n^{-c} \mid \mathscr{S}_{n}\right) \leq n^{-2} .
$$

On the other hand, we have (see [17], p. 543, Remark; the result therein states for the regular tree, but the same proof by convexity obviously holds in the general case)

$$
\mathbf{E}\left(\mathrm{e}^{-t \frac{\beta_{n}}{\mathbf{E}\left(\beta_{n}\right)}}\right) \leq \mathbf{E}\left(\mathrm{e}^{-t W_{n}}\right), \quad t \geq 0 .
$$

Since $\beta_{n}=0=W_{n}$ on $\mathscr{S}_{n}^{c}$, it is equivalent to say that $\mathbf{E}\left(\mathrm{e}^{-t \frac{\beta_{n}}{\mathbf{E}\left(\beta_{n}\right)}} \mid \mathscr{S}_{n}\right) \leq \mathbf{E}\left(\mathrm{e}^{-t W_{n}} \mid \mathscr{S}_{n}\right)$. Therefore, for any $\varepsilon>0$ and all sufficiently large $n$,

$$
\mathbf{P}\left(\frac{\beta_{n}}{\mathbf{E}\left(\beta_{n}\right)}<\mathrm{e}^{-\varepsilon n^{1 / 3}} \mid \mathscr{S}_{n}\right) \leq \mathrm{e}^{1} \mathbf{E}\left(\mathrm{e}^{-\mathrm{e}^{\varepsilon n^{1 / 3}} W_{n}} \mid \mathscr{S}_{n}\right) \leq n^{-2} \mathrm{e}+\mathrm{e}^{-n^{-c} \mathrm{e}^{\varepsilon n^{1 / 3}}} .
$$

Since $\mathscr{S} \subset \mathscr{S}_{n}$, this implies $\sum_{n} \mathbf{P}\left(\frac{\beta_{n}}{\mathbf{E}\left(\beta_{n}\right)}<\mathrm{e}^{-\varepsilon n^{1 / 3}} \mid \mathscr{S}\right) \leq \frac{1}{\mathbf{P}(\mathscr{S})} \sum_{n} \mathbf{P}\left(\frac{\beta_{n}}{\mathbf{E}\left(\beta_{n}\right)}<\mathrm{e}^{-\varepsilon n^{1 / 3}} \mid \mathscr{S}_{n}\right)<$ $\infty$. If (6.6) holds, then by the Borel-Cantelli lemma, on the set $\mathscr{S}, \mathbf{P}$-almost surely for all sufficiently large $n, \beta_{n} \geq \mathrm{e}^{-\varepsilon n^{1 / 3}} \mathbf{E}\left(\beta_{n}\right) \geq \exp \left\{-\left[2 \varepsilon+\left(\frac{3 \pi^{2} \sigma^{2}}{8}\right)^{1 / 3}\right] n^{1 / 3}\right\}$, and thus $\varrho_{n} \geq$ 
$\omega(\varnothing, \overleftarrow{\varnothing}) \exp \left\{-\left[2 \varepsilon+\left(\frac{3 \pi^{2} \sigma^{2}}{8}\right)^{1 / 3}\right] n^{1 / 3}\right\}$ (Lemma [5.1). In view of Fact 1.3, we obtain (6.5), the lower bound in Theorem 1.1.

The rest of the section is devoted to the proof of (6.6) . Let as before $\varrho_{n}:=P_{\omega}\left\{\tau_{n}<\tau_{0}\right\}$. Since $\beta_{n} \geq \varrho_{n}$ (Lemma 5.1 ), we only need to bound $\mathbf{E}\left(\varrho_{n}\right)$ from below.

For any vertex $x$, let $P_{\omega}^{x}$ be the (quenched) probability such that $P_{\omega}^{x}\left\{X_{0}=x\right\}=1$. We first prove a formula for $\varrho_{n}$ without the assumption $\psi(1)=\psi^{\prime}(1)=0$. We mention that if $|x|=n$, then under $P_{\omega}^{x}, \tau_{n}$ is the first return time to generation $n$.

Lemma 6.2 Assume that the walk $\left(X_{n}\right)$ is recurrent. For any $n \geq 1$, we have

$$
\varrho_{n}=\omega(\varnothing, \overleftarrow{\varnothing}) \sum_{|x|=n} \frac{\mathrm{e}^{-V(x)}}{\omega(x, \overleftarrow{x})} P_{\omega}^{x}\left\{\tau_{n}>\tau_{0}\right\}
$$

Proof of Lemma 6.2. The beginning of the proof uses a similar idea as in the proof of Lemma 5.1, except that instead of considering the number of returns to $\varnothing$ before hitting generation $n$, we consider the last site at generation $n$ visited by the walk during an excursion. More precisely, for any $x$ with $|x| \geq 1$, let $T_{x}^{0)}:=0$ and $T_{x}^{(k)}:=\inf \left\{i>T_{x}^{(k-1)}: X_{i}=x\right\}$ (for $k \geq 1$ ). In words, $T_{x}^{(k)}$ is the time of the $k$-th visit at $x$.

Recall that $\varrho_{n}$ is the (quenched) probability that during an excursion away from the root $\varnothing$, the walk hits generation $n$. By considering the last site at generation $n$ visited by the walk during the excursion, we have

$$
\begin{aligned}
\varrho_{n} & =\sum_{|x|=n} \sum_{k=1}^{\infty} P_{\omega}\left\{T_{x}^{(k)}<\tau_{0}<T_{x}^{(k+1)}, \max _{T_{x}^{(k)}<i \leq \tau_{0}}\left|X_{i}\right|<n\right\} \\
& =\sum_{|x|=n} \sum_{k=1}^{\infty} P_{\omega}\left\{T_{x}^{(k)}<\tau_{0}, \max _{T_{x}^{(k)}<i \leq \tau_{0}}\left|X_{i}\right|<n\right\} .
\end{aligned}
$$

Applying the strong Markov property at $T_{x}^{(k)}$, we see that the probability on the right-hand side equals $P_{\omega}\left\{T_{x}^{(k)}<\tau_{0}\right\} P_{\omega}^{x}\left\{\tau_{n}>\tau_{0}\right\}$. Therefore,

$$
\varrho_{n}=\sum_{|x|=n} P_{\omega}^{x}\left\{\tau_{n}>\tau_{0}\right\} \sum_{k=1}^{\infty} P_{\omega}\left\{T_{x}^{(k)}<\tau_{0}\right\}=\sum_{|x|=n} P_{\omega}^{x}\left\{\tau_{n}>\tau_{0}\right\} E_{\omega}\left(\sum_{i=0}^{\tau_{0}-1} \mathbf{1}_{\left\{X_{i}=x\right\}}\right) .
$$

$E_{\omega}\left(\sum_{i=0}^{\tau_{0}-1} \mathbf{1}_{\left\{X_{i}=x\right\}}\right)$, is the expected number of visits at site $x$ in an excursion, and can therefore be explicitly computed. Indeed one can easily check that, as a function of $x$, it is invariant with respect to the transition matrix $\omega(x, y)$. In the particular setting of Markov 
chains on trees any invariant measure can be computed, using an easy recurrence. One gets that all the invariant measures are proportional to $\pi(x):=\frac{1}{\omega(x, \overleftarrow{x})} \mathrm{e}^{-V(x)}$, for $x \neq \overleftarrow{\varnothing}$ (Note that this formula is also valid for $x=\varnothing$, because of the consistent definition of $\omega(\varnothing, \overleftarrow{\varnothing})$ ) Therefore, there exists $0<c(\omega)<\infty$ such that

$$
E_{\omega}\left(\sum_{i=0}^{\tau_{0}-1} \mathbf{1}_{\left\{X_{i}=x\right\}}\right)=\frac{c(\omega)}{\omega(x, \overleftarrow{x})} \mathrm{e}^{-V(x)}
$$

To determine the value of $c(\omega)$, we take $x:=\varnothing$, to see that $c(\omega)=\omega(\varnothing, \overleftarrow{\varnothing})$. This yields the lemma.

Assume $\psi(1)=0$. We make use of the size-biased branching random walk, and work under the new probability $\mathbf{Q}$. Recall the definitions of $\mathbf{Q}$ and $w_{n}$ from (6.1) and (6.2), respectively. By Lemma 6.2,

$$
\mathbf{E}\left(\varrho_{n}\right)=\mathbf{E}_{\mathbf{Q}}\left\{\frac{\omega(\varnothing, \overleftarrow{\varnothing})}{\omega\left(w_{n}, w_{n-1}\right)} P_{\omega}^{w_{n}}\left\{\tau_{n}>\tau_{0}\right\}\right\}
$$

We observe that

$$
P_{\omega}^{w_{n}}\left\{\tau_{n}>\tau_{0}\right\}=\prod_{j=1}^{n} P_{\omega}^{w_{j}}\left\{\tau_{n}>T\left(w_{j-1}\right)\right\}=: \prod_{j=1}^{n} Y_{j}
$$

Obviously, $Y_{n}=\omega\left(w_{n}, w_{n-1}\right), Y_{n-1}=\omega\left(w_{n-1}, w_{n-2}\right)$.

Let $j \leq n-2$. By the Markov property, $Y_{j}=\omega\left(w_{j}, w_{j-1}\right)+\sum_{x: \overleftarrow{x}=w_{j}} \omega\left(w_{j}, x\right) P_{\omega}^{x}\left\{\tau_{n}>\right.$ $\left.T\left(w_{j-1}\right)\right\}$, whereas by the strong Markov property, $P_{\omega}^{x}\left\{\tau_{n}>T\left(w_{j-1}\right)\right\}=P_{\omega}^{x}\left\{\tau_{n}>T\left(w_{j}\right)\right\} Y_{j}$ for all $x$ such that $\overleftarrow{x}=w_{j}$. Accordingly,

$$
Y_{j}=\frac{\omega\left(w_{j}, w_{j-1}\right)}{1-\sum_{x: \overleftarrow{x}=w_{j}} \omega\left(w_{j}, x\right) P_{\omega}^{x}\left\{\tau_{n}>T\left(w_{j}\right)\right\}}=\frac{1}{1+\sum_{x: \overleftarrow{x}=w_{j}} B(x) P_{\omega}^{x}\left\{\tau_{n}<T\left(w_{j}\right)\right\}}
$$

where

$$
B(x):=\mathrm{e}^{-[V(x)-V(\overleftarrow{x})]}=\frac{\omega(\overleftarrow{x}, x)}{\omega(\overleftarrow{x}, \overleftarrow{x})}
$$

So, if we write

$$
\xi_{j}:=\sum_{x: \overleftarrow{x}=w_{j}, x \neq w_{j+1}} B(x) P_{\omega}^{x}\left\{\tau_{n}<T\left(w_{j}\right)\right\}, \quad 1 \leq j \leq n-2
$$

then $Y_{j}=\frac{1}{1+\xi_{j}+\left(1-Y_{j+1}\right) B\left(w_{j+1}\right)}, 1 \leq j \leq n-2$, and $\mathbf{E}\left(\varrho_{n}\right)=\mathbf{E}_{\mathbf{Q}}\left\{\frac{\omega(\varnothing, \overleftarrow{\varnothing})}{\omega\left(w_{n}, w_{n-1}\right)} \prod_{j=1}^{n} Y_{j}\right\}=$ $\mathbf{E}_{\mathbf{Q}}\left\{\omega(\varnothing, \overleftarrow{\varnothing}) \prod_{j=1}^{n-1} Y_{j}\right\}$ 
Let $\mathscr{G}_{n}$ be the sigma-algebra generated by the first $n$ generations of the spine (see (6.4)). By Proposition 6.1, under $\mathbf{Q}$, the random variables $\xi_{1}, \cdots, \xi_{n-1}$ are conditionally independent given $\mathscr{G}_{n}$. Moreover, for any $1 \leq j \leq n-2$,

$$
\mathbf{E}_{\mathbf{Q}}\left(\xi_{j} \mid \mathscr{G}_{n}\right)=\sum_{x: \overleftarrow{x}=w_{j}, x \neq w_{j+1}} B(x) \mathbf{E}\left(\beta_{n-1-j}\right) \leq \frac{\mathbf{E}\left(\beta_{n-1-j}\right)}{\omega\left(w_{j}, w_{j-1}\right)} .
$$

We now provide a lower bound for $\mathbf{E}\left(\varrho_{n}\right)$, by replacing $\left(Y_{j}\right)_{1 \leq j \leq n-1}$ by a new collection of random variables, denoted by $\left(Z_{j}\right)_{1 \leq j \leq n-1}$ and defined as follows: $Z_{n-1}:=Y_{n-1}=$ $\omega\left(w_{n-1}, w_{n-2}\right)$ and for $1 \leq j \leq n-2$,

$$
Z_{j}:=\frac{1}{1+\mathbf{E}_{\mathbf{Q}}\left(\xi_{j} \mid \mathscr{G}_{n}\right)+\left(1-Z_{j+1}\right) B\left(w_{j+1}\right)} .
$$

Since $Z_{n-1}, B\left(w_{n-1}\right), B\left(w_{n-2}\right), \cdots, B\left(w_{2}\right)$ are $\mathscr{G}_{n}$-measurable, it follows by backwards induction on $j$ that each $Z_{j}$, for $1 \leq j \leq n-1$, is $\mathscr{G}_{n}$-measurable.

Lemma 6.3 Assume $\psi(1)=0$. For any $n \geq 3$, we have

$$
\mathbf{E}_{\mathbf{Q}}\left\{\prod_{j=1}^{n-1} Y_{j} \mid \mathscr{G}_{n}\right\} \geq \prod_{j=1}^{n-1} Z_{j}, \quad \text { Q-a.s. }
$$

Proof of Lemma 6.3. For any $c \in[0,1]$ and $a:=\left(a_{1}, \cdots, a_{n-1}\right) \in \mathbb{R}_{+}^{n-1}$, we define $F_{n-1}^{c, a}\left(u_{n-1}\right):=c, u_{n-1} \in \mathbb{R}_{+}$, and for $1 \leq j \leq n-2$,

$$
F_{j}^{c, a}\left(u_{j}, \cdots, u_{n-2}\right):=\frac{1}{1+u_{j}+a_{j+1}\left[1-F_{j+1}^{c, a}\left(u_{j+1}, \cdots, u_{n-2}\right)\right]}, \quad\left(u_{j}, \cdots, u_{n-2}\right) \in \mathbb{R}_{+}^{n-j-1} .
$$

Then by backwards induction on $j$, we have, for $1 \leq j \leq n-1$,

$$
Y_{j}=F_{j}^{Y_{n-1}, B(w)}\left(\xi_{j}, \cdots, \xi_{n-2}\right), \quad Z_{j}=F_{j}^{Z_{n-1}, B(w)}\left(\mathbf{E}_{\mathbf{Q}}\left(\xi_{j} \mid \mathscr{G}_{n}\right), \cdots, \mathbf{E}_{\mathbf{Q}}\left(\xi_{n-2} \mid \mathscr{G}_{n}\right)\right),
$$

where $B(w):=\left(B\left(w_{1}\right), \cdots, B\left(w_{n-1}\right)\right)$. Note that both $Y_{n-1}$ and $B(w)$ are $\mathscr{G}_{n}$-measurable.

Recall that (under Q) $\xi_{1}, \cdots, \xi_{n-2}$ are conditionally independent given $\mathscr{G}_{n}$. By Jensen's inequality, if $\Phi: \mathbb{R}_{+}^{n-2} \rightarrow \mathbb{R}$ is coordinate-wise convex, then $\left.\mathbf{E}_{\mathbf{Q}}\left\{\Phi\left(\xi_{1}, \cdots, \xi_{n-2}\right) \mid \mathscr{G}_{n}\right)\right\} \geq$ $\Phi\left(\mathbf{E}_{\mathbf{Q}}\left(\xi_{1} \mid \mathscr{G}_{n}\right), \cdots, \mathbf{E}_{\mathbf{Q}}\left(\xi_{n-2} \mid \mathscr{G}_{n}\right)\right)$, Q-a.s. So we only need to show that for any $c \in[0,1]$ and $a \in \mathbb{R}_{+}^{n-1},\left(u_{1}, \cdots, u_{n-2}\right) \mapsto \prod_{j=1}^{n-1} F_{j}^{c, a}\left(u_{j}, \cdots, u_{n-2}\right)$ as a function on $\mathbb{R}_{+}^{n-2}$, is convex in each of $u_{i}$.

Since the product of non-negative, coordinate-wise non-increasing, coordinate-wise convex functions is still (non-negative, coordinate-wise non-increasing, and) coordinate-wise 
convex, we only have to check that for any $j \leq n-2$, the function $\left(u_{j}, \cdots, u_{n-2}\right) \mapsto$ $F_{j}^{c, a}\left(u_{j}, \cdots, u_{n-2}\right)$ is non-negative (which is obvious), coordinate-wise non-increasing, and coordinate-wise convex. We prove it by induction on $j$.

By definition, $F_{n-2}^{c, a}\left(u_{n-2}\right)=\left[1+u_{n-2}+(1-c) a_{n-1}\right]^{-1}$, which is obviously non-increasing and convex in $u_{n-2}$.

Assume that for $1 \leq j \leq n-3,\left(u_{j+1}, \cdots, u_{n-2}\right) \mapsto F_{j+1}^{c, a}\left(u_{j+1}, \cdots, u_{n-2}\right)$ is coordinatewise non-increasing and coordinate-wise convex. Since

$$
F_{j}^{c, a}\left(u_{j}, \cdots, u_{n-2}\right)=\frac{1}{1+u_{j}+a_{j+1}\left[1-F_{j+1}^{c, a}\left(u_{j+1}, \cdots, u_{n-2}\right)\right]},
$$

$F_{j}^{c, a}$ is non-increasing and convex in each of $u_{i}$ (for $j \leq i \leq n-2$ ): the monotonicity is obvious, whereas the convexity follows from the fact that $y \mapsto \frac{1}{1+u_{j}+(1-y) a_{j+1}}$ is convex and non-decreasing on $[0,1]$ and that $f \circ g$ is convex if $f$ is convex and non-decreasing while $g$ is convex.

Recall that $\mathbf{E}\left(\varrho_{n}\right)=\mathbf{E}_{\mathbf{Q}}\left\{\omega(\varnothing, \overleftarrow{\varnothing}) \prod_{j=1}^{n-1} Y_{j}\right\}$. Since $\omega(\varnothing, \overleftarrow{\varnothing})$ is $\mathscr{G}_{n}$-measurable, it follows from Lemma 6.3 that

$$
\mathbf{E}\left(\varrho_{n}\right) \geq \mathbf{E}_{\mathbf{Q}}\left\{\omega(\varnothing, \overleftarrow{\varnothing}) \prod_{j=1}^{n-1} Z_{j}\right\}
$$

We now give a lower bound for $\prod_{j=1}^{n-1} Z_{j}$ by means of a deterministic lemma. The proof of the lemma is in the Appendix.

Lemma 6.4 Let $n>k \geq 2$. Let $b_{j+1}>0$ and $r_{j} \geq 0$ for all $0 \leq j<n$. Define $\left(z_{j}\right)_{1 \leq j \leq n}$ by $z_{n}=0$ and

$$
z_{j}:=\frac{1}{1+r_{j}+b_{j+1}\left(1-z_{j+1}\right)}, \quad 1 \leq j \leq n-1 .
$$

$\operatorname{Let} v(0):=0$ and $v(j):=-\sum_{i=1}^{j} \log b_{i}, 1 \leq j \leq n$. For any $m_{0}=0<m_{1}<\ldots<m_{k}=n-1$, we have

$$
\prod_{j=1}^{n-1} z_{j} \geq \frac{2^{-k}}{\prod_{i=1}^{k}\left(m_{i}-m_{i-1}\right)} \exp \left\{-\sum_{i=1}^{k}\left(\lambda_{i}+\left(m_{i}-m_{i-1}\right)^{2} r^{(i)} \mathrm{e}^{v_{i}^{*}}\right)\right\},
$$

where for $1 \leq i \leq k$ (with $y^{+}:=\max \{y, 0\}$ for $y \in \mathbb{R}$ ),

$$
\begin{aligned}
r^{(i)} & :=\max _{m_{i-1}<j \leq m_{i}} r_{j}, \\
\lambda_{i} & :=\max _{m_{i-1}<j \leq m_{i}}\left(v(j)-v\left(m_{i}\right)\right)+\left(v\left(m_{i}\right)-v\left(1+m_{i}\right)\right)^{+}, \\
v_{i}^{*} & :=\max _{m_{i-1}<j \leq \ell \leq m_{i}}(v(j)-v(\ell)) .
\end{aligned}
$$


We continue with the proof of the lower bound in Theorem 1.1. Recall from (6.9) that $\mathbf{E}\left(\varrho_{n}\right) \geq \mathbf{E}_{\mathbf{Q}}\left\{\omega(\varnothing, \overleftarrow{\varnothing}) \prod_{j=1}^{n-1} Z_{j}\right\}$

Let $k \geq 2$ and $m_{0}:=0<m_{1}<m_{2}<\ldots<m_{k}=n-1$. Taking $b_{j+1}=B\left(w_{j+1}\right)$ and $r_{j}:=\mathbf{E}_{\mathbf{Q}}\left(\xi_{j} \mid \mathscr{G}_{n}\right)$, we note from (6.8) that we may take the choice of $z_{j}=Z_{j}$ in Lemma 6.4. Applying this lemma, and arguing that $\prod_{i=1}^{k}\left(m_{i}-m_{i-1}\right) \leq \prod_{i=1}^{k} n=n^{k}$, we find that

$$
\mathbf{E}\left(\varrho_{n}\right) \geq \frac{1}{(2 n)^{k}} \mathbf{E}_{\mathbf{Q}}\left(\omega(\varnothing, \overleftarrow{\varnothing}) \mathrm{e}^{-\sum_{i=1}^{k} \Lambda_{i}-\sum_{i=1}^{k}\left(m_{i}-m_{i-1}\right)^{2} r^{(i)} \mathrm{e}^{S_{i}^{*}}}\right)
$$

where, for any $1 \leq i \leq k$,

$$
\begin{aligned}
r^{(i)} & :=\max _{m_{i-1}<j \leq m_{i}} \mathbf{E}_{\mathbf{Q}}\left(\xi_{j} \mid \mathscr{G}_{n}\right) \leq \max _{m_{i-1}<j \leq m_{i}} \frac{\mathbf{E}\left(\beta_{n-1-j}\right)}{\omega\left(w_{j}, w_{j-1}\right)}, \\
\Lambda_{i} & :=\max _{m_{i-1}<j \leq m_{i}}\left(S_{j}-S_{m_{i}}\right)+\left(S_{m_{i}}-S_{1+m_{i}}\right)^{+}, \\
S_{i}^{*} & :=\max _{m_{i-1}<j \leq \ell \leq m_{i}}\left(S_{j}-S_{\ell}\right),
\end{aligned}
$$

with $S_{j}:=V\left(w_{j}\right), 0 \leq j \leq n$. [In the inequality for $r^{(i)}$, we used (6.7).]

We choose: $\chi:=\frac{1}{100}, k:=\left\lfloor n^{\frac{1-\chi}{3}}\right\rfloor, m_{0}:=0, m_{i}:=n-(k-i)^{3}\left\lfloor n^{\chi}\right\rfloor$ for $1 \leq i \leq k-1$, and $m_{k}:=n-1$.

Let $c>1$ be a constant sufficiently large such that $\mathbf{Q}\left\{S_{2} \geq S_{1}, \omega\left(w_{1}, \varnothing\right) \geq \frac{1}{c}\right\}>\frac{1}{c}$. Let

$$
E_{n}^{(1)}:=\bigcap_{j=m_{k-1}+1}^{m_{k}}\left\{S_{j+1} \geq S_{j}, \omega\left(w_{j}, w_{j-1}\right) \geq \frac{1}{c}\right\} .
$$

On $E_{n}^{(1)}$, we have $\Lambda_{k} \leq 0, r^{(k)} \leq c$, and $S_{k}^{*}=0$, whereas by definition, $m_{k}-m_{k-1}=$ $\left\lfloor n^{\chi}\right\rfloor-1 \leq n^{\chi}$. Therefore, by (6.10),

$$
\begin{aligned}
\mathbf{E}\left(\varrho_{n}\right) & \geq \frac{\mathrm{e}^{-c n^{2 \chi}}}{(2 n)^{k}} \mathbf{E}_{\mathbf{Q}}\left(\omega(\varnothing, \overleftarrow{\varnothing}) \mathrm{e}^{-\sum_{i=1}^{k-1} \Lambda_{i}-\sum_{i=1}^{k-1}\left(m_{i}-m_{i-1}\right)^{2} r^{(i)} \mathrm{e}^{S_{i}^{*}}} \mathbf{1}_{E_{n}^{(1)}}\right) \\
& =\frac{\mathrm{e}^{-c n^{2 \chi}}}{(2 n)^{k}} \mathbf{E}_{\mathbf{Q}}\left(\omega(\varnothing, \overleftarrow{\varnothing}) \mathrm{e}^{-\sum_{i=1}^{k-1} \Lambda_{i}-\sum_{i=1}^{k-1}\left(m_{i}-m_{i-1}\right)^{2} r^{(i)} \mathrm{e}^{S_{i}^{*}}}\right) \mathbf{Q}\left(E_{n}^{(1)}\right)
\end{aligned}
$$

the last identity being a consequence of the fact (notation: $\left.w_{-1}:=\overleftarrow{\varnothing}\right)$ that under $\mathbf{Q},\left(S_{j}-\right.$ $S_{j-1}, \omega\left(w_{j-1}, w_{j-2}\right)$ ), for $j \geq 1$, are independent (they are i.i.d. for $j \geq 2$ ). By the definition of $c, \mathbf{Q}\left(E_{n}^{(1)}\right)=\left[\mathbf{Q}\left\{S_{2} \geq S_{1}, \omega\left(w_{1}, \varnothing\right) \geq \frac{1}{c}\right\}\right]^{m_{k}-m_{k-1}} \geq\left(\frac{1}{c}\right)^{m_{k}-m_{k-1}}=\left(\frac{1}{c}\right)^{\left\lfloor{ }^{\chi \chi}\right\rfloor-1}$. Hence,

$$
\mathbf{E}\left(\varrho_{n}\right) \geq \frac{\mathrm{e}^{-c n^{2 \chi}}}{(2 n)^{k} c^{\left\lfloor n^{\chi}\right\rfloor-1}} \mathbf{E}_{\mathbf{Q}}\left(\omega(\varnothing, \overleftarrow{\varnothing}) \mathrm{e}^{-\sum_{i=1}^{k-1} \Lambda_{i}-\sum_{i=1}^{k-1}\left(m_{i}-m_{i-1}\right)^{2} r^{(i)} \mathrm{e}^{S_{i}^{*}}}\right)
$$


Let $\varepsilon \in\left(0, \frac{\chi}{3}\right)$. Write $a_{*}:=\left(\frac{3 \pi^{2} \sigma^{2}}{8}\right)^{1 / 3}$. By (5.4), there exists some constant $c_{1}>0$ such that $\mathbf{E}\left(\beta_{i}\right) \leq c_{1} \mathrm{e}^{-\left(a_{*}-\varepsilon\right)(i+1)^{1 / 3}}$ for all $i \geq 1$. Thus

$$
r^{(i)} \leq \frac{c_{1} \mathrm{e}^{-\left(a_{*}-\varepsilon\right)\left(n-m_{i}\right)^{1 / 3}}}{\min _{m_{i-1}<j \leq m_{i}} \omega\left(w_{j}, w_{j-1}\right)}, \quad 1 \leq i \leq k .
$$

Consider

$$
E_{n}^{(2)}:=\left\{\omega\left(w_{j}, w_{j-1}\right) \geq \mathrm{e}^{-n^{\varepsilon}}, \forall 1 \leq j \leq m_{k-1}\right\} \cap\left\{\omega(\varnothing, \overleftarrow{\varnothing}) \geq \mathrm{e}^{-n^{\varepsilon}}\right\}
$$

On $E_{n}^{(2)}$, we have, for any $1 \leq i \leq k-1, r^{(i)} \leq c_{1} \mathrm{e}^{-\left(a_{*}-\varepsilon\right)\left(n-m_{i}\right)^{1 / 3}+n^{\varepsilon}}$, whereas $m_{i}-m_{i-1} \leq n$, thus $\left(m_{i}-m_{i-1}\right)^{2} r^{(i)} \leq \mathrm{e}^{-\left(a_{*}-2 \varepsilon\right)\left(n-m_{i}\right)^{1 / 3}}$ (for all sufficiently large $n$; we insist on the fact that $i<k)$. Hence

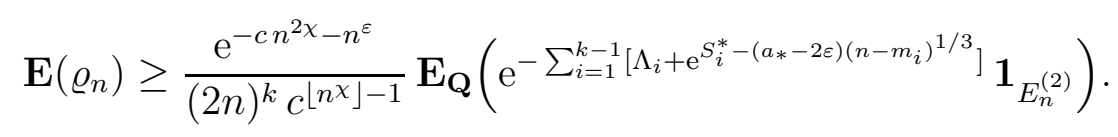

Let, for $1 \leq i \leq k-1$,

$$
E_{n, i}^{(3)}:=\left\{S_{i}^{*}<\left(a_{*}-2 \varepsilon\right)\left(n-m_{i}\right)^{1 / 3}, \max _{m_{i-1}<j \leq m_{i}}\left(S_{j}-S_{m_{i}}\right) \leq n^{\varepsilon},\left|S_{1+m_{i}}-S_{m_{i}}\right| \leq n^{\varepsilon}\right\} .
$$

On the event $E_{n, i}^{(3)}$ (for $1 \leq i \leq k-1$ ), we have $\Lambda_{i} \leq n^{\varepsilon}+n^{\varepsilon}=2 n^{\varepsilon}$, and, of course, $S_{i}^{*}-\left(a_{*}-2 \varepsilon\right)\left(n-m_{i}\right)^{1 / 3} \leq 0$, so that $\Lambda_{i}+\mathrm{e}^{S_{i}^{*}-\left(a_{*}-2 \varepsilon\right)\left(n-m_{i}\right)^{1 / 3}} \leq 2 n^{\varepsilon}+1 \leq 3 n^{\varepsilon}$. Going back to (6.11), we obtain:

$$
\mathbf{E}\left(\varrho_{n}\right) \geq \frac{\mathrm{e}^{-c n^{2 \chi}-n^{\varepsilon}-3 n^{\varepsilon}(k-1)}}{\left.(2 n)^{k} c^{\lfloor\chi\rfloor}\right\rfloor} \mathbf{Q}\left(E_{n}^{(2)} \cap \bigcap_{i=1}^{k-1} E_{n, i}^{(3)}\right) .
$$

By independence,

$$
\begin{aligned}
\mathrm{Q}\left(E_{n}^{(2)} \cap \bigcap_{i=1}^{k-1} E_{n, i}^{(3)}\right) & =\mathbf{Q}\left\{\omega(\varnothing, \overleftarrow{\varnothing}) \geq \mathrm{e}^{-n^{\varepsilon}}\right\} \prod_{i=1}^{k-1} \mathrm{Q}\left(E_{n, i}^{(3)}, \min _{m_{i-1}<\ell \leq m_{i}} \omega\left(w_{\ell}, w_{\ell-1}\right) \geq \mathrm{e}^{-n^{\varepsilon}}\right) \\
& \geq \frac{1}{2} \prod_{i=1}^{k-1} \mathrm{Q}\left(E_{n, i}^{(3)}, \min _{m_{i-1}<\ell \leq m_{i}} \omega\left(w_{\ell}, w_{\ell-1}\right) \geq \mathrm{e}^{-n^{\varepsilon}}\right)
\end{aligned}
$$

the last inequality holding for all sufficiently large $n$ (in view of the fact that $\mathbf{Q}\{\omega(\varnothing, \overleftarrow{\varnothing}) \geq$ 
$\left.\left.\mathrm{e}^{-n^{\varepsilon}}\right\} \rightarrow 1, n \rightarrow \infty\right)$. By independence again, for any $1 \leq i \leq k-1$,

$$
\begin{aligned}
& \mathbf{Q}\left(E_{n, i}^{(3)}, \min _{m_{i-1}<\ell \leq m_{i}} \omega\left(w_{\ell}, w_{\ell-1}\right) \geq \mathrm{e}^{-n^{\varepsilon}}\right) \\
= & \mathbf{Q}\left(\left|S_{1+m_{i}}-S_{m_{i}}\right| \leq n^{\varepsilon}, \omega\left(w_{m_{i}}, w_{m_{i}-1}\right) \geq \mathrm{e}^{-n^{\varepsilon}}\right) \times \\
& \times \mathbf{Q}\left(S_{i}^{*}<\left(a_{*}-2 \varepsilon\right)\left(n-m_{i}\right)^{1 / 3}, \max _{m_{i-1}<j \leq m_{i}}\left(S_{j}-S_{m_{i}}\right) \leq n^{\varepsilon},\right. \\
& \left.\min _{m_{i-1}<\ell<m_{i}} \omega\left(w_{\ell}, w_{\ell-1}\right) \geq \mathrm{e}^{-n^{\varepsilon}}\right) \\
= & \mathbf{Q}\left(\left|S_{2}-S_{1}\right| \leq n^{\varepsilon}, \omega\left(w_{1}, \varnothing\right) \geq \mathrm{e}^{-n^{\varepsilon}}\right) \times \mathbf{Q}\left(F_{i}(n), \min _{1 \leq \ell<\Delta_{i}} \omega\left(w_{\ell}, w_{\ell-1}\right) \geq \mathrm{e}^{-n^{\varepsilon}}\right),
\end{aligned}
$$

where, for $1 \leq i \leq k-1$,

$$
\begin{aligned}
\Delta_{i} & :=m_{i}-m_{i-1}, \\
F_{i}(n) & :=\left\{\max _{1 \leq \ell \leq \Delta_{i}}\left(\bar{S}_{\ell}-S_{\ell}\right)<\left(a_{*}-2 \varepsilon\right)\left(n-m_{i}\right)^{1 / 3}, \bar{S}_{\Delta_{i}}-S_{\Delta_{i}} \leq n^{\varepsilon}\right\}
\end{aligned}
$$

with $\bar{S}_{\ell}:=\max _{1 \leq j \leq \ell} S_{j}$ as before. Again, $\mathbf{Q}\left\{\left|S_{2}-S_{1}\right| \leq n^{\varepsilon}, \omega\left(w_{1}, \varnothing\right) \geq \mathrm{e}^{-n^{\varepsilon}}\right\}$ is greater than $\frac{1}{2}$ for large $n$ because it converges to 1 . Therefore, for all large $n$,

$$
\mathbf{Q}\left(E_{n}^{(2)} \cap \bigcap_{i=1}^{k-1} E_{n, i}^{(3)}\right) \geq \frac{1}{2^{k}} \prod_{i=1}^{k-1} \mathbf{Q}\left(F_{i}(n), \min _{1 \leq \ell<\Delta_{i}} \omega\left(w_{\ell}, w_{\ell-1}\right) \geq \mathrm{e}^{-n^{\varepsilon}}\right) .
$$

To bound the probability expression on the right-hand side, we use the following lemma, which is a uniform version of Proposition 3.1. Its proof is in the Appendix.

Lemma 6.5 Let $S_{i}-S_{i-1}, i \geq 1$, be i.i.d. mean-zero random variables $\left(S_{0}:=0\right)$ with $\sigma^{2}:=\mathbf{E}\left(S_{1}^{2}\right) \in(0, \infty)$. For any $\delta>0$, there exist $r_{0}>1$ and $0<\eta<1$ such that for all $r \in\left[r_{0}, \eta \sqrt{n}\right] \cap \mathbb{Z}$, for all events $A_{i}^{(n)}, 1 \leq i \leq n$, satisfying the following two conditions:

- $\left(S_{i}-S_{i-1}, A_{i}^{(n)}\right)$, for $1 \leq i \leq n$, are i.i.d.,

- $\mathbf{P}\left(\cap_{i=1}^{n} A_{i}^{(n)}\right) \geq 1-\frac{\eta}{r}$,

we have

$$
\frac{\eta}{r} \mathrm{e}^{-(1+\delta) \frac{\pi^{2} \sigma^{2}}{8} \frac{n}{r^{2}}} \leq \mathbf{P}\left(\max _{1 \leq i \leq n}\left(\bar{S}_{i}-S_{i}\right)<r, \bar{S}_{n}=S_{n}, \bigcap_{i=1}^{n} A_{i}^{(n)}\right) \leq \mathrm{e}^{-(1-\delta) \frac{\pi^{2} \sigma^{2}}{8} \frac{n}{r^{2}}}
$$

where $\bar{S}_{i}:=\max _{1 \leq j \leq i} S_{j}$.

Recall that $\left(A_{1}, \cdots, A_{N}\right)$ is a random vector distributed as any of $\left(A_{1}(x), \cdots, A_{N(x)}(x)\right)$ defined in (1.1). Since $\mathbf{E}\left[\frac{1}{\omega(\varnothing, \overleftarrow{\varnothing})}\right]=\mathbf{E}\left[1+\sum_{i=1}^{N} A_{i}\right]=1+\mathrm{e}^{\psi(1)}=2$, we have, $\mathbf{Q}\left\{\omega\left(w_{1}, \varnothing\right)<\right.$ 
$\left.\mathrm{e}^{-n^{\varepsilon}}\right\}=\mathbf{P}\left\{\omega(\varnothing, \overleftarrow{\varnothing})<\mathrm{e}^{-n^{\varepsilon}}\right\} \leq 2 \mathrm{e}^{-n^{\varepsilon}}$ (by Markov's inequality). Therefore, for all large $n$ and all $1 \leq i \leq k-1$,

$$
\begin{aligned}
\mathbf{Q}\left\{\min _{1 \leq \ell<\Delta_{i}} \omega\left(w_{\ell}, w_{\ell-1}\right) \geq \mathrm{e}^{-n^{\varepsilon}}\right\} & =\left[\mathbf{Q}\left\{\omega\left(w_{1}, \varnothing\right) \geq \mathrm{e}^{-n^{\varepsilon}}\right\}\right]^{\Delta_{i}-1} \\
& \geq\left(1-2 \mathrm{e}^{-n^{\varepsilon}}\right)^{\Delta_{i}-1} \\
& \geq\left(1-2 \mathrm{e}^{-n^{\varepsilon}}\right)^{n} \geq 1-\mathrm{e}^{-n^{\varepsilon / 2}} .
\end{aligned}
$$

We apply Lemma 6.5 to $A_{i}^{(n)}:=\left\{\omega\left(w_{i}, w_{i-1}\right) \geq \mathrm{e}^{-n^{\varepsilon}}\right\}\left(\right.$ with $\Delta_{i}-1$ and $\left(a_{*}-2 \varepsilon\right)\left(n-m_{i}\right)^{1 / 3}$ playing the roles of $n$ and $r$, respectively; noting that $\Delta_{i}-1 \sim 3(k-i)^{2} n^{\chi}$ and $\left(n-m_{i}\right)^{1 / 3} \sim$ $(k-i) n^{\chi / 3}$, so the last condition in the lemma on $A_{i}^{(n)}$ is satisfied), to see that for all large $n$ and for all $1 \leq i \leq k-1$,

$$
\mathrm{Q}\left(F_{i}(n), \min _{1 \leq \ell<\Delta_{i}} \omega\left(w_{\ell}, w_{\ell-1}\right) \geq \mathrm{e}^{-n^{\varepsilon}}\right) \geq \exp \left(-(1+\varepsilon) \frac{3 \pi^{2} \sigma^{2}}{8\left(a_{*}-2 \varepsilon\right)^{2}} n^{\chi / 3}\right),
$$

which implies that

$$
\mathrm{Q}\left(E_{n}^{(2)} \cap \bigcap_{i=1}^{k-1} E_{n, i}^{(3)}\right) \geq \frac{1}{2^{k}} \exp \left(-(k-1)(1+\varepsilon) \frac{3 \pi^{2} \sigma^{2}}{8\left(a_{*}-2 \varepsilon\right)^{2}} n^{\chi / 3}\right) .
$$

By definition, $k=\left\lfloor n^{(1-\chi) / 3}\right\rfloor$; hence

$$
\liminf _{n \rightarrow \infty} \frac{1}{n^{1 / 3}} \log \mathbf{Q}\left(E_{n}^{(2)} \cap \bigcap_{i=1}^{k-1} E_{n, i}^{(3)}\right) \geq-(1+\varepsilon) \frac{3 \pi^{2} \sigma^{2}}{8\left(a_{*}-2 \varepsilon\right)^{2}} .
$$

This, together with (6.12), yields

$$
\liminf _{n \rightarrow \infty} \frac{1}{n^{1 / 3}} \log \mathbf{E}\left(\varrho_{n}\right) \geq-\left(\frac{3 \pi^{2} \sigma^{2}}{8}\right)^{1 / 3} .
$$

Since $\beta_{n} \geq \varrho_{n}$ (Lemma 5.1), we obtain (6.6), thus the lower bound in Theorem 1.1.

\section{A Appendix. Proofs of Lemmas 6.4 and 6.5}

Proof of Lemma 6.4. Although the lemma is deterministic, our proof is probabilistic. We note that the value of $b_{1}$ plays no role in the lemma.

Let $\left(\eta_{i}\right)_{i \geq 0}$ be a Markov chain on $\{0,1, \cdots, n\}$ with transition probabilities

$$
\mathbf{P}\left(\eta_{i+1}=k \mid \eta_{i}=j\right)= \begin{cases}\frac{b_{j+1}}{1+b_{j+1}}, & \text { if } k=j+1, \\ \frac{1}{1+b_{j+1}}, & \text { if } k=j-1 .\end{cases}
$$


[The transition probabilities from $j=0$ and $j=n$, having no importance, can be anything. For example, we can take $\mathbf{P}\left\{\eta_{i+1}=0 \mid \eta_{i}=0\right\}=1=\mathbf{P}\left\{\eta_{i+1}=n \mid \eta_{i}=n\right\}$.] Define $\tau_{\eta}(j):=\inf \left\{i \geq 1: \eta_{i}=j\right\}$ (with $\inf \varnothing:=\infty$ as usual). Let $\mathbf{P}_{j}$ be the probability such that $\mathbf{P}_{j}\left\{\eta_{0}=j\right\}=1$, and let $\mathbf{E}_{j}$ be the expectation with respect to $\mathbf{P}_{j}$. We claim that ( $\infty$ being not $<\infty)$

$$
\prod_{j=1}^{n-1} z_{j} \geq \prod_{i=1}^{k} \mathbf{E}_{m_{i}}\left(\mathbf{1}_{\left(\tau_{\eta}\left(m_{i-1}\right)<\tau_{\eta}\left(m_{i}\right)\right)}\left(1+r^{(i)}\right)^{-\tau_{\eta}\left(m_{i-1}\right)}\right)
$$

and for any integers $0 \leq \ell<m \leq n$ and $r \geq 0$,

$$
\begin{aligned}
\mathbf{E}_{m}\left((1+r)^{-\tau_{\eta}(\ell)} \mathbf{1}_{\left(\tau_{\eta}(\ell)<\tau_{\eta}(m)\right)}\right) & \geq \frac{1}{2(m-\ell)} \exp \left\{-\max _{\ell<i \leq m}(v(i)-v(m))\right. \\
-(v(m) & \left.-v(m+1))^{+}-r(m-\ell)^{2} \mathrm{e}^{\max _{\ell<i \leq j \leq m}[v(i)-v(j)]}\right\} .
\end{aligned}
$$

Plainly Lemma 6.4 will follow from (A.1) and (A.2).

To prove (A.1), we consider a Markov chain $\left(\widetilde{\eta}_{i}\right)_{i \geq 0}$ on $\{0,1, \cdots, n\} \cup\{\partial\}$, where $\partial$ is an absorbing point, such that

$$
\mathbf{P}\left(\widetilde{\eta}_{i+1}=k \mid \widetilde{\eta}_{i}=j\right)=\left\{\begin{array}{ll}
b_{j+1} q_{j}, & \text { if } k=j+1, \\
q_{j}, & \text { if } k=j-1, \\
r_{j} q_{j}, & \text { if } k=\partial,
\end{array} \quad 0<j<n,\right.
$$

with $q_{j}:=\frac{1}{b_{j+1}+1+r_{j}}$ for all $0<j<n$. [Again, the transition probabilities from $j=0$ and $j=n$ can be anything.] Let $\tau_{\tilde{\eta}}(j):=\inf \left\{i \geq 1: \widetilde{\eta}_{i}=j\right\}$. Then

$$
z_{j}=\mathbf{P}_{j}\left(\tau_{\widetilde{\eta}}(j-1)<\tau_{\widetilde{\eta}}(n)\right), \quad \forall 1 \leq j \leq n-1 .
$$

Let us check (A.3): $z_{n-1}=q_{n-1}$, and if $z_{j}=\mathbf{P}_{j}\left(\tau_{\tilde{\eta}}(j-1)<\tau_{\tilde{\eta}}(n)\right)$ for $j \in[2, n-1] \cap \mathbb{Z}$, then $\mathbf{P}_{j-1}\left(\tau_{\tilde{\eta}}(j-2)<\tau_{\tilde{\eta}}(n)\right)=q_{j-1}+b_{j} q_{j-1} \mathbf{P}_{j}\left(\tau_{\tilde{\eta}}(j-2)<\tau_{\tilde{\eta}}(n)\right)=q_{j-1}+b_{j} q_{j-1} z_{j} \mathbf{P}_{j-1}\left(\tau_{\tilde{\eta}}(j-2)<\right.$ $\left.\tau_{\widetilde{\eta}}(n)\right)$ by the Markov property. Hence $\mathbf{P}_{j-1}\left(\tau_{\tilde{\eta}}(j-2)<\tau_{\widetilde{\eta}}(n)\right)=\frac{q_{j-1}}{1-b_{j} q_{j-1} z_{j}}$, which, by the definition of $q_{j-1}$, is $\frac{1}{1+r_{j-1}+b_{j}\left(1-z_{j}\right)}$. Hence $\mathbf{P}_{j-1}\left(\tau_{\widetilde{\eta}}(j-2)<\tau_{\widetilde{\eta}}(n)\right)=z_{j-1}$. This establishes (A.3). As a consequence,

$$
\mathbf{P}_{n-1}\left(\tau_{\widetilde{\eta}}(0)<\tau_{\widetilde{\eta}}(n)\right)=\prod_{j=1}^{n-1} z_{j} .
$$

We claim that for $0 \leq \ell<m<n$,

$$
\mathbf{P}_{m}\left(\tau_{\widetilde{\eta}}(\ell)<\tau_{\widetilde{\eta}}(m)\right) \geq \mathbf{E}_{m}\left(\mathbf{1}_{\left(\tau_{\eta}(\ell)<\tau_{\eta}(m)\right)}(1+r)^{-\tau_{\eta}(\ell)}\right),
$$


where $r:=\max _{\ell<j \leq m} r_{j}$. Since $\mathbf{P}_{n-1}\left(\tau_{\tilde{\eta}}(0)<\tau_{\tilde{\eta}}(n)\right) \geq \prod_{i=1}^{k} \mathbf{P}_{m_{i}}\left(\tau_{\tilde{\eta}}\left(m_{i-1}\right)<\tau_{\widetilde{\eta}}\left(m_{i}\right)\right)$, (A.1) will be a consequence of (A.4) and (A.5).

To prove (A.5), let $\Omega_{\ell, m}$ be the set of all (finite) paths of $\widetilde{\eta}$ starting from $m$ and hitting $\ell$ before returning to $m$ (and without being absorbed by $\partial$ ). For any $\gamma \in \Omega_{\ell, m}$, let $L_{\gamma}^{ \pm}(j):=$ $\sum_{i \geq 0} \mathbf{1}_{\left(\gamma_{i}=j, \gamma_{i+1}=j \pm 1\right)}$ and $L_{\gamma}(j):=L_{\gamma}^{+}(j)+L_{\gamma}^{-}(j)$. Then

$$
\begin{aligned}
\mathbf{P}_{m}\left(\tau_{\tilde{\eta}}(\ell)<\tau_{\tilde{\eta}}(m)\right) & =\sum_{\gamma \in \Omega_{\ell, m}} \prod_{\ell<j \leq m}\left(b_{j+1} q_{j}\right)^{L_{\gamma}^{+}(j)}\left(q_{j}\right)^{L_{\gamma}^{-}(j)} \\
& =\sum_{\gamma \in \Omega_{\ell, m}} \prod_{\ell<j \leq m}\left(\frac{b_{j+1}}{1+b_{j+1}}\right)^{L_{\gamma}^{+}(j)}\left(\frac{1}{1+b_{j+1}}\right)^{L_{\gamma}^{-}(j)}\left(\frac{1+b_{j+1}}{1+b_{j+1}+r_{j}}\right)^{L_{\gamma}(j)} \\
& \geq \sum_{\gamma \in \Omega_{\ell, m}} \prod_{\ell<j \leq m}\left(\frac{b_{j+1}}{1+b_{j+1}}\right)^{L_{\gamma}^{+}(j)}\left(\frac{1}{1+b_{j+1}}\right)^{L_{\gamma}^{-}(j)}\left(\frac{1}{1+r}\right)^{L_{\gamma}(j)} \\
& =\mathbf{E}_{m}\left(\mathbf{1}_{\left(\tau_{\eta}(\ell)<\tau_{\eta}(m)\right)}(1+r)^{-\tau_{\eta}(\ell)}\right),
\end{aligned}
$$

yielding (A.5) and hence (A.1).

It remains to show (A.2). We note that $\mathbf{P}_{m}\left\{\tau_{\eta}(\ell)<\tau_{\eta}(m)\right\}=\frac{1}{1+b_{m+1}} \mathbf{P}_{m-1}\left\{\tau_{\eta}(\ell)<\right.$ $\left.\tau_{\eta}(m)\right\}$. To compute $\mathbf{P}_{m-1}\left\{\tau_{\eta}(\ell)<\tau_{\eta}(m)\right\}$ for the birth-and-death chain, we recall $v(j)=$ $-\sum_{i=1}^{j} \log b_{i}$ for $j \geq 1$, and use the same argument as in the second identity of (1.10) to see that $\mathbf{P}_{m-1}\left\{\tau_{\eta}(\ell)<\tau_{\eta}(m)\right\}=\frac{\mathrm{e}^{v(m)}}{\sum_{i=\ell+1}^{m} \mathrm{e}^{v(i)}}$. Therefore,

$$
\begin{aligned}
\mathbf{P}_{m}\left(\tau_{\eta}(\ell)<\tau_{\eta}(m)\right) & =\frac{1}{1+b_{m+1}} \frac{\mathrm{e}^{v(m)}}{\sum_{i=\ell+1}^{m} \mathrm{e}^{v(i)}} \\
& \geq \frac{1}{2(m-\ell)} \exp \left\{-\max _{\ell<i \leq m}(v(i)-v(m))-(v(m)-v(m+1))^{+}\right\} .
\end{aligned}
$$

Under $\mathbf{P}_{m}$ and conditionally on $\left\{\tau_{\eta}(\ell)<\tau_{\eta}(m)\right\}, \tau_{\eta}(\ell)$ is stochastically smaller than the hitting time of $\ell$ by a Markov chain with the same probability transition as $\eta$ but reflecting on $m$. The expectation of the latter hitting time was estimated by Golosov ([14], p. 498, (A.1)). Hence

$$
\mathbf{E}_{m}\left(\tau_{\eta}(\ell) \mid \tau_{\eta}(\ell)<\tau_{\eta}(m)\right) \leq(m-\ell)^{2} \exp \left(\max _{\ell<i \leq j \leq m}(v(i)-v(j))\right),
$$

which, by means of the elementary inequality $(1+r)^{-u} \geq \mathrm{e}^{-r u}$ for $u \geq 0$ and Jensen's inequality, implies that

$$
\mathbf{E}_{m}\left((1+r)^{-\tau_{\eta}(\ell)} \mid \tau_{\eta}(\ell)<\tau_{\eta}(m)\right) \geq \exp \left(-r(m-\ell)^{2} \mathrm{e}^{\max _{\ell<i \leq j \leq m}(v(i)-v(j))}\right) .
$$


This together with (A.6) implies (A.2), completing the proof of Lemma 6.4.

Proof of Lemma 6.5. We start with the proof of the lower bound. Let $a \geq 2$ be an integer whose value will be chosen later on. Let $\eta<\frac{1}{a}$. Let $K:=\left\lfloor\frac{n}{a r^{2}}\right\rfloor$, and $n_{i}=i a r^{2}$ for $0 \leq i \leq K-1$ and $n_{K}:=n$. Write $S_{n}^{\#}:=\max _{1 \leq i \leq n}\left(\bar{S}_{i}-S_{i}\right)$. It is clear that

$$
\begin{aligned}
& \mathbf{P}\left(S_{n}^{\#}<r, \bar{S}_{n}=S_{n}, \bigcap_{i=1}^{n} A_{i}^{(n)}\right) \\
\geq & \mathbf{P}\left(\forall 1 \leq j \leq K, \max _{n_{j-1}<i \leq n_{j}}\left(\bar{S}_{i}-S_{i}\right)<r, \bar{S}_{n_{j}}-S_{n_{j}}<\delta r, \bar{S}_{n}=S_{n}, \bigcap_{i=1}^{n} A_{i}^{(n)}\right) .
\end{aligned}
$$

For any $1 \leq j \leq K$, conditionally on $\sigma\left\{S_{i}, A_{i}^{(n)}, 1 \leq i \leq n_{j-1}\right\}$ and on $\left\{\bar{S}_{n_{j-1}}-S_{n_{j-1}}=x\right\}$, the reflecting random walk $\left(\bar{S}_{i+n_{j-1}}-S_{i+n_{j-1}}, 0 \leq i \leq n_{j}-n_{j-1}\right)$ has the same law as $\left(\max \left\{x, \bar{S}_{i}\right\}-S_{i}, 0 \leq i \leq n_{j}-n_{j-1}\right)$. Accordingly,

$$
\mathbf{P}\left(S_{n}^{\#}<r, \bar{S}_{n}=S_{n}, \bigcap_{i=1}^{n} A_{i}^{(n)}\right) \geq q_{n, r}^{K-1} b_{n, r}
$$

where

$$
\begin{aligned}
& q_{n, r}:=\mathbf{P}\left(S_{a r^{2}}^{\#}<(1-\delta) r, \bar{S}_{a r^{2}}-S_{a r^{2}}<\delta r, \bar{S}_{a r^{2}}>\delta r, \bigcap_{i=1}^{a r^{2}} A_{i}^{(n)}\right), \\
& b_{n, r}:=\mathbf{P}\left(S_{n_{K}-n_{K-1}}^{\#}<(1-\delta) r, \bar{S}_{n_{K}-n_{K-1}}=S_{n_{K}-n_{K-1}}>\delta r, \bigcap_{i=1}^{n_{K}-n_{K-1}} A_{i}^{(n)}\right) .
\end{aligned}
$$

We observe that

$$
\begin{aligned}
q_{n, r} & \geq \mathbf{P}\left(S_{a r^{2}}^{\#}<(1-\delta) r, \bar{S}_{a r^{2}}-S_{a r^{2}}<\delta r, \bar{S}_{a r^{2}}>\delta r\right)+\mathbf{P}\left(\bigcap_{i=1}^{a r^{2}} A_{i}^{(n)}\right)-1 \\
& \geq \mathbf{P}\left(S_{a r^{2}}^{\#}<(1-\delta) r, \bar{S}_{a r^{2}}-S_{a r^{2}}<\delta r, \bar{S}_{a r^{2}}>\delta r\right)-\frac{\eta}{r} .
\end{aligned}
$$

On the other hand, since the three events $\left\{S_{a r^{2}}^{\#}<(1-\delta) r\right\},\left\{\bar{S}_{a r^{2}}-S_{a r^{2}}<\delta r\right\}$ and $\left\{\bar{S}_{a r^{2}}>\delta r\right\}$ are non-decreasing with respect to each $S_{i}-S_{i-1}$ (for $1 \leq i \leq n$ ), it follows from the FKG inequality that

$$
q_{n, r} \geq \mathbf{P}\left(S_{a r^{2}}^{\#}<(1-\delta) r\right) \mathbf{P}\left(\bar{S}_{a r^{2}}-S_{a r^{2}}<\delta r\right) \mathbf{P}\left(\bar{S}_{a r^{2}}>\delta r\right)-\frac{\eta}{r} .
$$


By Donsker's invariance principle,

$$
\begin{aligned}
& \lim _{r \rightarrow \infty} \mathbf{P}\left(S_{a r^{2}}^{\#}<(1-\delta) r\right) \mathbf{P}\left(\bar{S}_{a r^{2}}-S_{a r^{2}}<\delta r\right) \mathbf{P}\left(\bar{S}_{a r^{2}}>\delta r\right) \\
= & \mathbf{P}\left(\sup _{t \in[0,1]}(\bar{W}(t)-W(t))<\frac{1-\delta}{\sigma \sqrt{a}}\right) \mathbf{P}\left(\bar{W}(1)-W(1)<\frac{\delta}{\sigma \sqrt{a}}\right) \mathbf{P}\left(\bar{W}(1)>\frac{\delta}{\sigma \sqrt{a}}\right),
\end{aligned}
$$

where $W$ is a standard Brownian motion, with $\bar{W}(t):=\sup _{s \in[0, t]} W(s)$ for any $t$. Recall that $\sup _{t \in[0,1]}(\bar{W}(t)-W(t))$ has the same distribution as $\sup _{t \in[0,1]}|W(t)|$; so (see Formula (5.9), page 342 of Feller [12]) $\mathbf{P}\left\{\sup _{t \in[0,1]}(\bar{W}(t)-W(t)) \leq x\right\}=\exp \left\{-(1+o(1)) \frac{\pi^{2}}{8 x^{2}}\right\}$, for $x \rightarrow 0$. Consequently, for all sufficiently large $a$, say $a \geq a_{0}$, we have

$$
\mathbf{P}\left(\sup _{t \in[0,1]}(\bar{W}(t)-W(t))<\frac{1-\delta}{\sigma \sqrt{a}}\right) \geq \mathrm{e}^{-(1+3 \delta) \frac{\pi^{2} \sigma^{2} a}{8}} .
$$

Since both $\bar{W}(1)-W(1)$ and $\bar{W}(1)$ are distributed as the absolute value of a standard Gaussian random variable, we can even increase the value of $a_{0}$ (if necessary) such that for all $a \geq a_{0}$ (thus $\frac{\delta}{\sigma \sqrt{a}}$ is very small), $\mathbf{P}\left\{\bar{W}(1)>\frac{\delta}{\sigma \sqrt{a}}\right\} \geq \frac{1}{2}$ and $\mathbf{P}\left\{\bar{W}(1)-W(1)<\frac{\delta}{\sigma \sqrt{a}}\right\} \geq \frac{\delta}{2 \sigma \sqrt{a}}$.

Now we fix an arbitrary integer $a \geq a_{0}$. For all large $r$ (say $r \geq r_{0}$ ) such that $r \leq \eta \sqrt{n}$, we have

$$
q_{n, r} \geq \mathrm{e}^{-(1+4 \delta) \frac{\pi^{2} \sigma^{2} a}{8}} .
$$

The probability $b_{n, r}$ can be estimated in a similar way: from the assumptions on $\left(A_{i}^{(n)}\right)$ and the FKG inequality, we deduce that

$$
b_{n, r} \geq \mathbf{P}\left(S_{n_{K}-n_{K-1}}^{\#}<(1-\delta) r\right) \mathbf{P}\left(\bar{S}_{n_{K}-n_{K-1}}=S_{n_{K}-n_{K-1}}\right) \mathbf{P}\left(\bar{S}_{n_{K}-n_{K-1}}>\delta r\right)-\frac{\eta}{r} .
$$

Observe that $a r^{2} \leq n_{K}-n_{K-1} \leq 2 a r^{2}$. Therefore, by Donsker's invariance principle, for all $r \geq r_{0}$ (with an increased value of $r_{0}$ if necessary), $\mathbf{P}\left(S_{n_{K}-n_{K-1}}^{\#}<(1-\delta) r\right) \mathbf{P}\left(\bar{S}_{n_{K}-n_{K-1}}>\right.$ $\delta r) \geq c(a, \delta)$ for some constant $c(a, \delta)>0$, whereas $\mathbf{P}\left(\bar{S}_{n_{K}-n_{K-1}}=S_{n_{K}-n_{K-1}}\right)=\mathbf{P}\left(S_{1} \geq\right.$ $\left.0, S_{2} \geq 0, \ldots, S_{n_{K}-n_{K-1}-1} \geq 0\right) \geq \frac{c^{\prime}}{r \sqrt{a}}$ for some constant $c^{\prime}>0$. Taking $\eta:=\min \left\{\frac{c(a, \delta) c^{\prime}}{2 \sqrt{a}}, \frac{1}{a}\right\}$, we get $b_{n, r} \geq \frac{\eta}{r}$. Consequently,

$$
\mathbf{P}\left(S_{n}^{\#}<r, \bar{S}_{n}=S_{n}, \bigcap_{i=1}^{n} A_{i}^{(n)}\right) \geq q_{n, r}^{K-1} b_{n, r} \geq \frac{\eta}{r} \mathrm{e}^{-(1+4 \delta) \frac{\pi^{2} \sigma^{2}}{8} \frac{n}{r^{2}}}
$$

proving the lower bound in the lemma.

The upper bound is easier: with the same notation and the choice of $a$, we have

$$
\begin{aligned}
\mathbf{P}\left(S_{n}^{\#}<r, \bar{S}_{n}=S_{n}, \bigcap_{i=1}^{n} A_{i}^{(n)}\right) & \leq \mathbf{P}\left(\forall 1 \leq j \leq K-1, \max _{n_{j-1}<i \leq n_{j}}\left(\bar{S}_{i}-S_{i}\right)<r\right) \\
& \leq\left[\mathbf{P}\left(S_{a r^{2}}^{\#}<r\right)\right]^{K-1} .
\end{aligned}
$$


For all large $r, \mathbf{P}\left(S_{a r^{2}}^{\#}<r\right) \leq \mathrm{e}^{-(1-\delta) \frac{\pi^{2} \sigma^{2} a}{8}}$; hence $\mathbf{P}\left(S_{n}^{\#}<r, \bar{S}_{n}=S_{n}, \cap_{i=1}^{n} A_{i}^{(n)}\right) \leq$ $\mathrm{e}^{-(1-\delta) \frac{\pi^{2} \sigma^{2}}{8} \frac{n}{r^{2}}-1}$, yielding the upper bound (by eventually modifying the choice of $\eta$ in terms of $a)$.

\section{Acknowledgement}

We have been kindly informed by Ofer Zeitouni that Theorem 1.4 in the case of regular trees was independently proved by Fang and Zeitouni [10]. We are also grateful to an anonymous referee for her/his very careful reading of the original manuscript.

\section{References}

[1] Aidékon, E. (2008). Transient random walks in random environment on a GaltonWatson tree. Probab. Theory Related Fields 142, 525-559.

[2] Aidékon, E. (2010). Large deviations for transient random walks in random environment on a Galton-Watson tree. Ann. Inst. H. Poincaré Probab. Statist. 46, 159-189.

[3] Ben Arous, G., Fribergh, A., Gantert, N. and Hammond, A. (2007+). Biased random walks on a Galton-Watson tree with leaves. ArXiv math.PR/0711.3686

[4] Ben Arous, G. and Hammond, A. (2009+). Stable limit laws for randomly biased random walks on trees. (in preparation)

[5] Biggins, J.D. (1976). The first- and last-birth problems for a multitype age-dependent branching process. Adv. Appl. Probab. 8, 446-459.

[6] Biggins, J.D. (1977). Chernoff's theorem in the branching random walk. J. Appl. Probab. 14, 630-636.

[7] Biggins, J.D. and Kyprianou, A.E. (1997). Seneta-Heyde norming in the branching random walk. Ann. Probab. 25, 337-360.

[8] Billingsley, P. (1968). Convergence of Probability Measures. Wiley, New York.

[9] Borodin, A.N. and Salminen, P. (2002). Handbook of Brownian motion - Facts and Formulae. Second edition. Birkhäuser, Basel.

[10] Fang, M. and Zeitouni, O. (2010). Consistent minimal displacement of branching random walks. Electron. Commun. Probab. 15, 106-118.

[11] Faraud, G. (2011). A central limit theorem for random walk in a random environment on marked Galton-Watson trees. Electron. Journ. Probab. 16, 174-215. 
[12] Feller, William (1971). An introduction to probability and its applications. Vol II. Second edition. John Wiley \& sons, Inc. New York-London-Sydney.

[13] Gantert, N., Hu, Y. and Shi, Z. (2008+). Asymptotics for the survival probability in a killed branching random walk. ArXiv math.PR/0811.0262

[14] Golosov, A.O. (1984). Localization of random walks in one-dimensional random environments. Comm. Math. Phys. 92, 491-506.

[15] Harris, T.E. (1963). The Theory of Branching Processes. Springer, Berlin.

[16] Hu, Y. and Shi, Z. (1997). Extreme lengths in Brownian and Bessel excursions. Bernoulli 3, 387-402.

[17] Hu, Y. and Shi, Z. (2007). A subdiffusive behaviour of recurrent random walk in random environment on a regular tree. Probab. Theory Related Fields 138, 521-549.

[18] Hu, Y. and Shi, Z. (2007). Slow movement of recurrent random walk in random environment on a regular tree. Ann. Probab. 35, 1978-1997.

[19] Hu, Y. and Shi, Z. (2009). Minimal position and critical martingale convergence in branching random walks, and directed polymers on disordered trees. Ann. Probab. 37, $742-789$.

[20] Jaffuel, B. (2009+). The critical barrier for the survival of the branching random walk with absorption. ArXiv math.PR/0911.2227

[21] Kahane, J.-P. and Peyrière, J. (1976). Sur certaines martingales de Mandelbrot. Adv. Math. 22, 131-145.

[22] Lyons, R. (1990). Random walks and percolation on trees. Ann. Probab. 18, 931-958.

[23] Lyons, R. (1992). Random walks, capacity and percolation on trees. Ann. Probab. 20, 2043-2088.

[24] Lyons, R. (1997). A simple path to Biggins' martingale convergence for branching random walk. In: Classical and Modern Branching Processes (Eds.: K.B. Athreya and P. Jagers). IMA Volumes in Mathematics and its Applications 84, 217-221. Springer, New York.

[25] Lyons, R. and Pemantle, R. (1992). Random walk in a random environment and firstpassage percolation on trees. Ann. Probab. 20, 125-136.

[26] Lyons, R., Pemantle, R. and Peres, Y. (1995). Conceptual proofs of $L \log L$ criteria for mean behavior of branching processes. Ann. Probab. 23, 1125-1138. 
[27] Lyons, R., Pemantle, R. and Peres, Y. (1995). Ergodic theory on Galton-Watson trees: speed of random walk and dimension of harmonic measure. Ergodic Theory Dynam. Systems 15, 593-619.

[28] Lyons, R., Pemantle, R. and Peres, Y. (1996). Biased random walks on Galton-Watson trees. Probab. Theory Related Fields 106, 249-264.

[29] McDiarmid, C. (1995). Minimal positions in a branching random walk. Ann. Appl. Probab. 5, 128-139.

[30] Menshikov, M.V. and Petritis, D. (2002). On random walks in random environment on trees and their relationship with multiplicative chaos. In: Mathematics and Computer Science II (Versailles, 2002), pp. 415-422. Birkhäuser, Basel.

[31] Mogulskii, A.A. (1974). Small deviations in the space of trajectories. Theory Probab. Appl. 19, 726-736.

[32] Peres, Y. and Zeitouni, O. (2008). A central limit theorem for biased random walks on Galton-Watson trees. Probab. Theory Related Fields 140, 595-629.

[33] Sinai, Ya.G. (1982). The limiting behavior of a one-dimensional random walk in a random medium. Th. Probab. Appl. 27, 256-268.

[34] Zeitouni, O. (2004). Random Walks in Random Environment, XXXI summer school in probability, St Flour (2001), Lecture Notes in Math. 1837, pp. 193-312. Springer, Berlin, 2004.

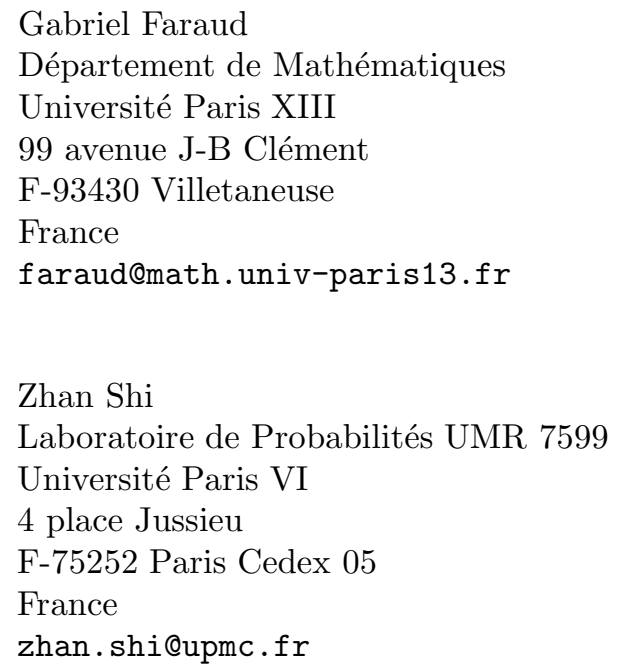

Yueyun $\mathrm{Hu}$

Département de Mathématiques

Université Paris XIII

99 avenue J-B Clément

F-93430 Villetaneuse

France

yueyun@math . univ-paris13.fr 